\title{
UN QUARTIER ROMAIN DE BEAUVAIS (Oise)
}

\author{
par Pierre LEMAN
}

Important carrefour routier, Beaurais a souffert considérablement des bombardements de juin 1940 qui ont partiellement anéanti la ville médiévale. Au chevet de la cathédrale, les ruines des maisons incendiées furent arasées et, dès les années cinquante, on y envisagea d'y installer la Galerie nationale de la Tapisserie. Ce projet connut plusieurs avatars. Le financement fit défaut et les plans présentés n’eurent point les accords désirés. En 1966, II. le Professeur WVill, alors J)irecteur de la circonscription Nord-Picardie, nous demanda d'ouvrir quelques tranchées de sondages dans l'angle nord-ouest dlu réduit fortifié du Bas-Empire (fig. 1, nos 12 et 26 ).

C"est en cet endroit en effet que la muraille du Bas-Empire marque un retour vers l'est selon un angle droit renforcé par une tour carrée. Des terrains ainsi enclos par la muraille romaine et où s'élevaient jadis les maisons ranoniales à l'ombre de l'imposant chœur gothique de la cathédrale, les archéologues ne connaissaient absolument rien. Il ne fallait donc pas manquer l'occasion. $A$ ce moment-la nous avons dì interrompre quelque temps la fouille de l'abbaye Saint-Lucien ${ }^{1}$ pour ouvrir deux sondages. l'un au pied du rempart, l'autre un peu plus loin, Loujours dans l'enclos de la maison de la Belle Image. En 1967, les choses se précisent et il convient de profiter au maximum de ce terrain ainsi libéré de toute construction avant l'arrivée des engins chargés de préparer les fondations de la Galerie de la Tapisserie (qui remplaçait la manufacture).

Trois grands sondages furent ouverts alors en juillet et aoùt 1967, a fin de couvrir au mieux la surface de lemprise du futur batiment. Les fouilles allaient bon train quand nous apprimes que le projet, cependant bien avancie, etait encore modifié. En hâte un nouveau programme de fouilles fut mis sur pied et on ouvrit un nouveau sondage. L'ampleur des vestiges fut telle que se posa le probleme de leur conservation. Il fallut se résigner alors à préparer un projet de galerie qui lui tiendrait compte des vestiges, de leur conservation et de leur présentation. Les campagnes de 1968 et de 1969 furent consacrées à explorer le

1 Voir notre article : l.es fonilles de l'Abhaye Suint-Lucien-de-Beaubais campanne 1966, dans Cahiers de Picardie, 1977, n" 1, p. $277-288$. 
plus grand secteur compris, en concertation cette fois avec MM. Jouve et Hermant, architectes chargés du projet, définitif cette fois². Voici l'essentiol des découvertes effectuées sur le chantier de 1966 a $19699^{3}$, seulement pour les périodes romaines.

Capitale de la Civilas des Bellovaques, l'antique Caesaromagus est beaucoup moins bien connue que sa consœur Samarobriva, plus au nord. Il nest pas de notre propos de refaire l'historiographie archéologique de Beauvais, mais a fin de mieux situer les vestiges découverts au chevet de la rathédrale, nous proposons un plan sommaire des découvertes de Beauvais depuis le Xvile siècle. Cette ville est construite sur une terrasse de sables grossiers qui s'étalent sur la pente douce qui descend vers la rivière Lo Thérain qui coule d'ouest en est. La rive sud de la rivière est dominée par un brusque talus de craie qui barre l'horizon de toute la ville sur une hauteur d'une trentaine de mètres tandis qu'au nord, à l'est et à l'ouest les terrains remontent légèrement en paliers successifs vers le plateau picard (fig. $\mathbf{2}$ ).

Seuls les documents routiers mentionnent le nom antique de Beauvais, Caesaromagus, sans oublier toutefois une citation chez Ptolémée. Le plan fig. 3 montre l'emplacement des. fouilles ouvertes en 1966 au pied des vestiges de la maison dite de la Belle Image, à louest de la cathédrale (fig. 1, no 12). Un ensemble thermal fut mis au jour pendant l'hiver 19011902, au cours duquel l'architecte Acher, entreprit des travaux de restauration à l'église Saint-Étienne. Il s'ensuivit des fouilles à l'intérieur mème de l'eglise ct une surface totale de 445 $\mathrm{m}^{2}$ fut ainsi explorée. Dans ce secteur, il n'y eut jamais de fouille, mème lors de la reconstruction du quartier en 1950)-1960, et il est done impossible de restituer ces thermes dans le tissu urbain antique . Premier édifice antique connu a Beauvais, le temple du mont Capron a été découvert au norl de la ville au $\mathrm{xvII}^{\mathrm{e}}$ s. A ce temple et son péripole, étaient jointes une terrasse et une esplanade carrée que réunissait un escalier ${ }^{6}$.

En juillet 1959, XII. Iemaire et Chami ont relevé une importante stratigraphie au cours de la destruction d'une butte artificielle située entre les rues Racine, Ricard, NicolasPasteur et Saint-Laurent?. Quelques années plus Lard, M. Jurvin a pu relever le plan d'une habitation romaine ainsi que le tracé d'une rue entre les rues du loocteur (iérard et Biot ${ }^{8}$. C'est au même chercheur que l'on doit la découverte d'un autre habitat, au coin de la rue

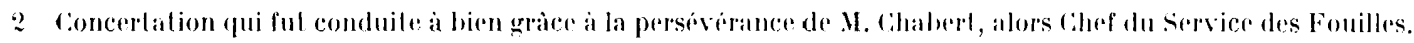

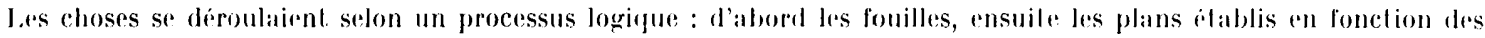
decouvertes et de leur conservation, enfin la consleuction proprement dite. Sur place, M. Blanchet. fut pour nous un precieux informaleur. ()u’il soit ici remercié.

3 Ces fouilles ont livere des masses considerables de ceramiques du Moyen Agre. Nous rn avons donne un apereu sommare dans un article paru dans Archéologie . Wédiévale II, 1972, La córamique peinte du Voyen Age déconerte à Bealuvais, p. $187-200$.

4 Itineraire d'Antonin, Table de Peutinger ; Porésés, fiéggraphie, II, 94.

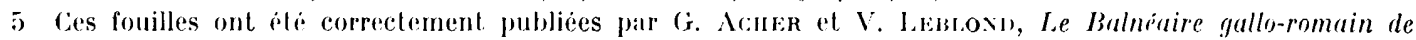
Beauvais, Caen, 1906, .I. Paquel, archilecte des Monuments historiques, nous assure n'avoir rien déconvert lors de sondages entrepris dans le chour de Saint-Etienne en 1961.

6 le plan publie par Victor Leblond, l'historien de Beauvais, a cte commente par . G. Grixirr, Manuel III, p. 416-419. Joseph Paulus a rassemblé toute da documentation relative a la lite des Bellovagnes pour un mémoire de licence présenté à l'Liniversité de Liegge en 1971.

7 A. PIGAxior, Informalions archéologiques, dans Gallia, XIX, 1961, p. 301.

8 Jierre 1)trvix, lin coup de sonde à travers les vestiges gallo-romains de Carsaromagus, dans $O g a m, \mathrm{XV}, 1963$, p. 49-64. 
Saint-Pantaléon et de lavenue Foch". Quant aux néropoles, dès le xvire siècle, elles ont livré un matériel particulièrement riche, in inscriptions notamment. La plus vaste et. peut-ître la plus ancienne, sétendait vers le nord-est. a proximite de la prison actuelle, le long de la voie romaine Beauvais-Saint-laléry ${ }^{10}$. Au surl, le long de la route BeauvaisParis, une autre nécropole. moins étendure, a éti explorée des $1841^{11}$. Enfin, au nord-ouest, rue du Pressoir Coquet, ancienne portion de la voie romaine Beauvais-Bavay, existe une petite nécropole dont la fondation doit dater du Bas-Empire ${ }^{12}$.

Tel nous apparaissait l'état de la cuestion lorsque nous abordames le problème des fouilles en 1966. L'essentiel di nos connaissances se résumait done pour une grande part aux données de l'étude du I)r Leblond au début de ce siècle, à laquelle il fallait ajonter les observations de lapres-guerre réunies par MII. Vasselle. Lemaire, Chami, Iurvin et Cartier ${ }^{13}$. Ces chercheurs ont eu le mérite de sauver ce qui pouvait l'ètre, avec hélas des moyens dérisoires. Valgré l’étendue des terrains libérés par les destructions dues aux bombardements de 1940, aucune grande fouille d'envergure ne fut entreprise comme cela le fut de facon prescue systématique dans les villes allemandes. De cette capitale de la cite des Bellovaques, nous ne connaissions, a part le temple du mont Capron, ni l'emplacement du forum, ni les principaux monuments, ni de facon générale, les grands axes de son plan l'ensimble.

De 1966 a 1969, dans l'angle nord-est du réduit forlifié du Bas-Empire, 30 ouvertures de sol furent pratiqueses a fin d'exploration arrhéologique selon des profondeurs et des surfaces variables. Chaque campagne a vu des numérotations différentes et non suivies étant donné les incertitudes des projets de la Cialerie nationale de la Tapisserie. Ainsi le sondlage 1 de 1966 n'est plus celui de 1967 et encore moins celui de 1968. Pour simplifier les choses, nous proposons le plan numéroté comme suit (fig. 1) :

1 (juillet 1967) ouverture d'un profond sondage - fouille d'une maison romaine et de sa cave,

2 (juillet 1968) recherche des niveaux romains apres défonçage de caves modernes,

3 (juillet 1968 ) fouille de la place romaine,

A (aout 1968) fouille d'une maison médievale installée sur des niveaux du Bas-Empire et du Haut Moyen Age,

5) (juillet 1967) fouille en dessous de l'ancienne rue de l'Mbbé Gellée.

if (aout 1967) fouille d'une maison romaine ef d'un lit de pierres romaines.

Les numéros de 1 à 6 correspondent au somdage 1 des années 1968 a 1969 .

7 (aoùt 1969) exploralion d'une grande cave médievale el d'un puits (sondage I., 1969),

8 (septembre 1969) recherche entre les $n^{\circ} 7$ el 1.1 (ex. sondage :)/1968), sondage $V$,

9 (juillet et aoùt 1969 ) sondage I, 1, I, 2.

10 idem (tranchee ouverte en vue de la recherche de la partie sud de la place romaine).

9) Gallia, XXI, 1963, p. 368-369.

10 Cette nécopole mérilerait une etude grobale. Ine home présentation chez l'historien de Beaurais : V. Lebloxu, La topographie romaine de Beauvais el son enceinle an IVe siecle, dans Bull. Arch. Comité travaux hisloriques, 1915 , p. 3,39 , p. 22 .

11 V. LeBlown, La lopographe..., op. cil., p. 24.

12 Voir le travial de J. el F. CARTIE, l.e cimeliere gallo-romain a inhumalion de la rue du Pressoir-Coquel, dans Revue du Nord, n" 195, 1967, p. 691-702.

13 Voir la nole d'A. Pigaviof, Informalions archéolngiques, dans Giallia, XVII, 1959, p. 282-283. 


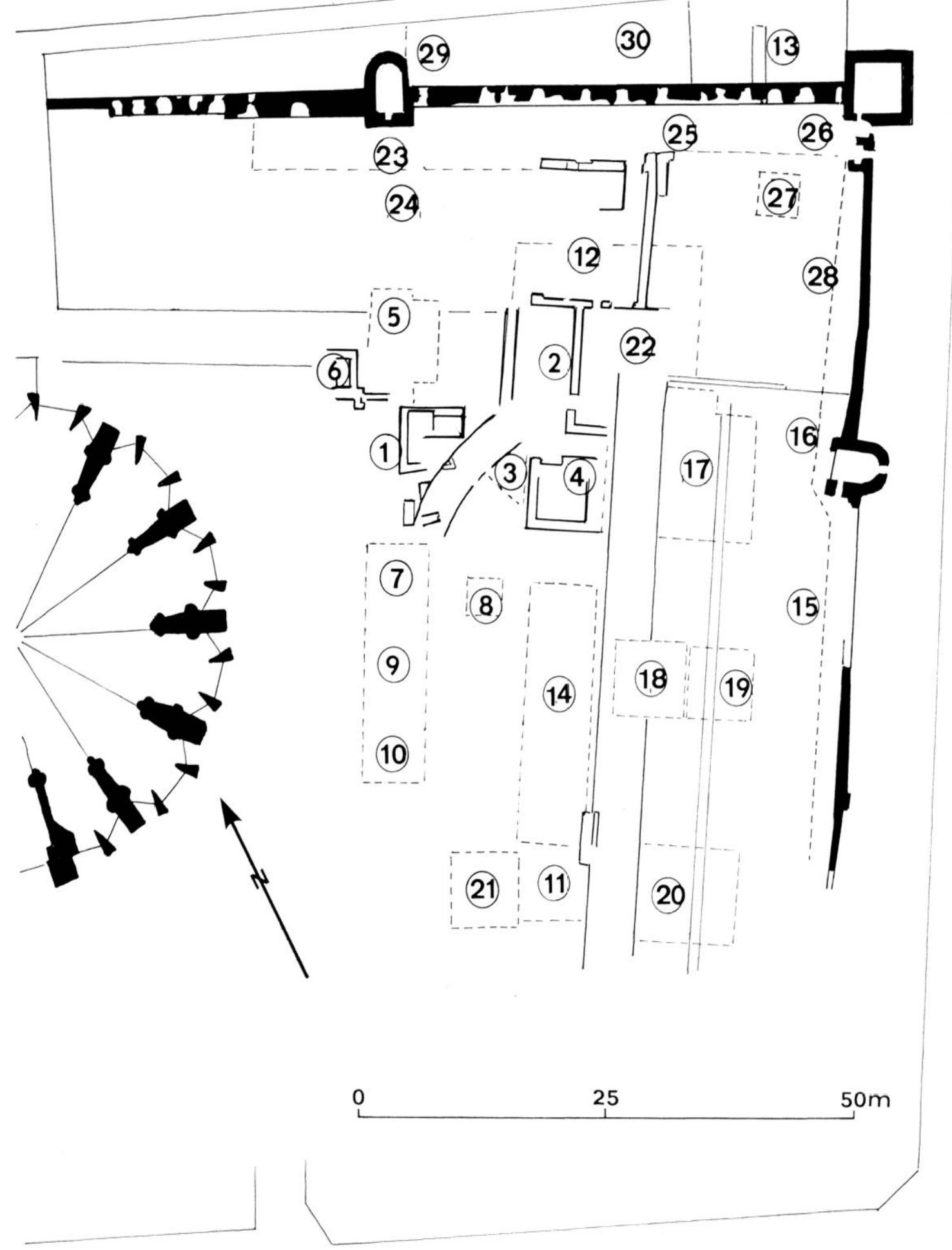

1 Emplacement des sondages ol carres de fouilles ouverts de 1966 a 1969. 
11 prolongation en septembre 1965) vers le sud du sondage 14 (ex. sondage j) de 1968),

1: Tranchée de fouilles ouvertes en 1966 au pied de l'escalier de la maison de la Belle Image, alin de rechercher le niveau romain,

1:) grande tranchée ouverte en 1969 perpendiculaire au rempart du caslrum, afin d'en rechercher le niveau des fondations,

1.1 grand sondage ouvert en 1969 dans la prolongation de 11.

15) Iranchée ouverte en 1969, afin d'étudier la face interne du rempart romain,

16 exploration de la cave creusée sous la lour romaine,

17 grand sondage ouvert en juillet 1968 - fouille de la rue romaine,

18 sondage ouvert en 1967 en rue de rechercher les assises profondes de la rue de l'Abbé Gellée,

19 sondage complémentaire au premier ouvert en 1968 .

20 sondage, entrant dans le mème programme que les deux précédents, ouvert en 1968.

21 sondage ouvert en 1967 alin de rechercher la branche sud de l'exiodre,

22 apres la destruction et l'arasement des vestiges de la Belle Image, recherche des niveaux romains (sondage I) et annexes en 1969).

$2: 3$ sondagre $B$ de la campagne 1969 an pied de la tour saint-Hilaire,

24 prolongation du précédent vers le sud (sondage 1 de 1969).

25 exploration des caves des maisons canoniales (1967. 1968. 1969).

2 ; relevé de la tranchée de fondation interne du rempart (1967),

27 sondage profond ouvert en 1967.

28 mème opération que le sondage 26 en 1969 (sondages F 9, H, I, J),

29 sondage externe au pierl de la tour saint-Milaire,

:30 exploration des caves implantées sous les jardins silues rue Racine.

\section{Les rules.}

Sur le plan du I)r Leblond ${ }^{14}$ figurent plusieurs tracés de rues romaines, réclles ou supposécs. I a rue Saint-Pierre y est mentionnée comme une section de la grande voie estouest. Elle reliait les deux portes est et ouest du caslrum du Bas-Empire, celle du Chatel l'une part, du Limaçon d'autre part. Nous avons pu mettre au jour des vestiges de deux rues au cours des sondages $20,18,19,17,26,13$ et 25, pour la rue nord-sud, et des sondages 23 et 24 pour la rue est-ouest.

La rue nord-sud mesurait dans sa plus grande largeur li) m au moins. Nous lavons repérée sur $90 \mathrm{~m}$ de long. I)u nord vers le sud, elle se présente comme ceci : en 1966, le sondage $1: 3$ avait révélé en son fond au niveau 6.).70 un revetement de silex, tandis qu'en 1969) un sonrlage limité rlans une cave médiévale mettait au jour, et selon la même cote. également le mème niveau de silex (sondage 2) $)$. Ia distance entre ces deux niveaux de silex donne cette largeur de 15) m, a l'exclusion des fossés toutefois.

Ia mème rue a été écalement repérée dans les sondages 20 de 1968 el 18 de 1967.1 )ans ce dernier. l'exploration a pu être menée de telle sorte que la stratigraphie de la voie et de ses recharges ait pu atre hien relevée. On retiendra donc que cette voie est composée de plusieurs assises de silex roulés, recouverte d'une couche de terres grises mêlées a du sable hlane. Ie premier niveau de silex sètend au niveau $64 \mathrm{~m}$, le dernier dans sa plus grande largeur a $6.5,50 \mathrm{~m}$. I.a roupe relevée appelle la comparaison immédiate avec celle étudiéc

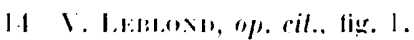




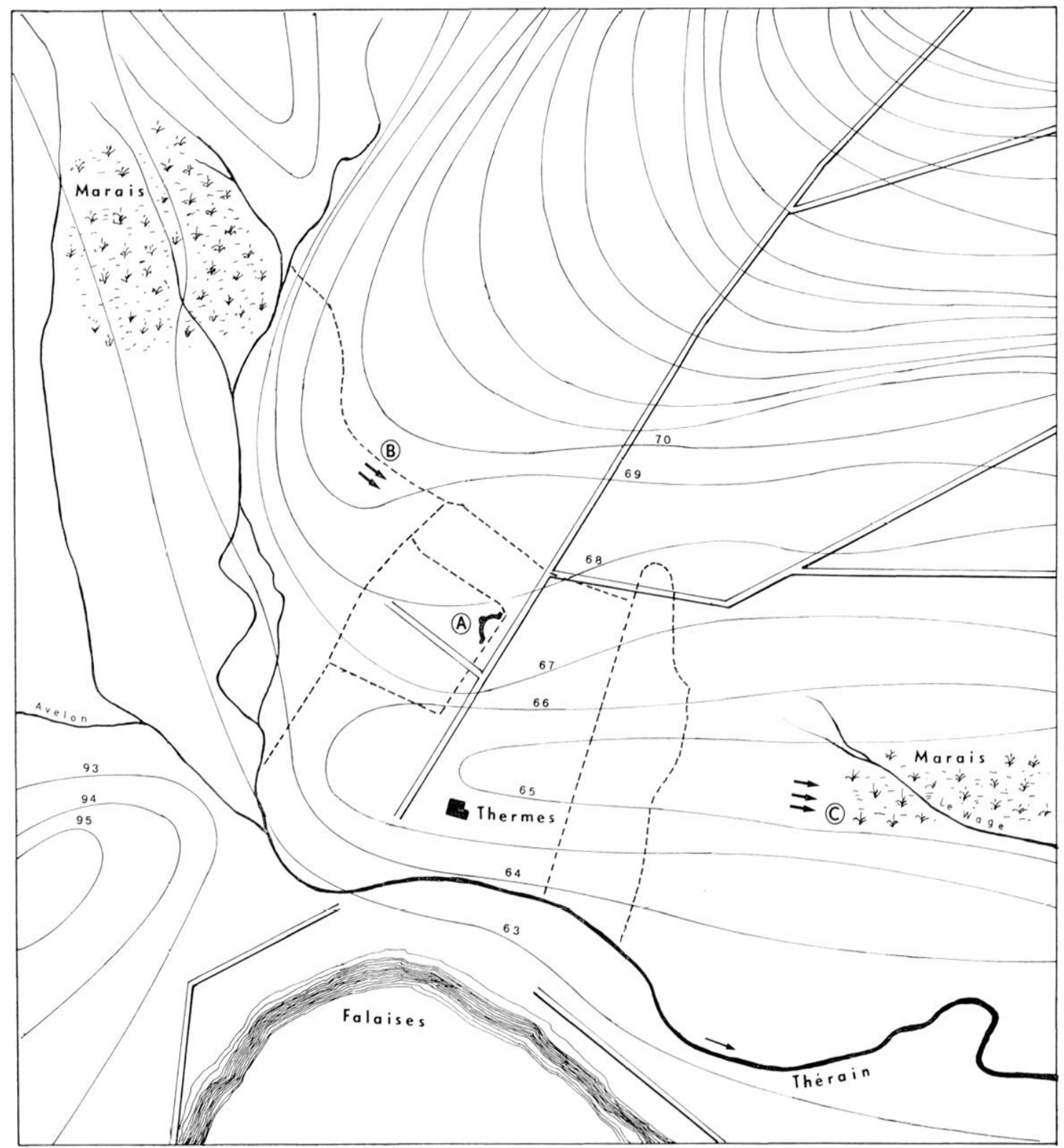

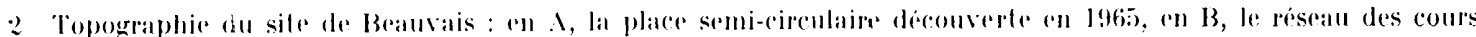

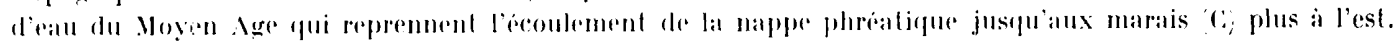

par lirancois Vasselle à Amiens systime identique de recharge. epandage de terres argileuses, absence de dallage ${ }^{15}$.

Le fossé n’a été repéré que sur le flanc oriental. Il mesurait près l'un mètre de large sur $1.50 \mathrm{~m}$ de profondeur. Comme partout ailleurs dans ce secteur, la voie a été installéc sur un épais matelas de craie concassée, laquelle repose directement sur une masse de boue

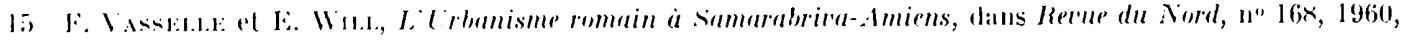

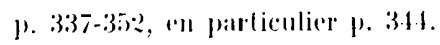




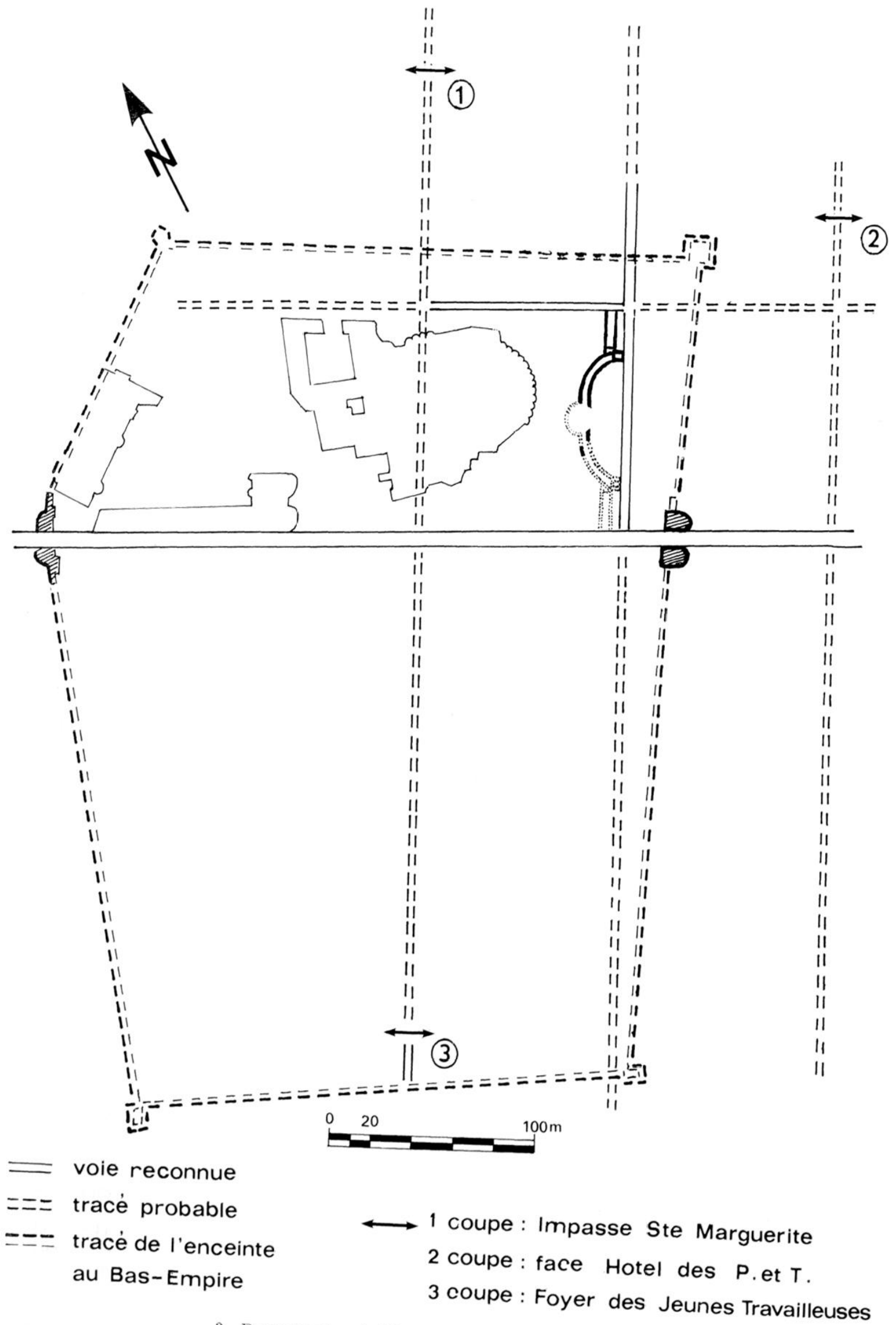

3 Reconstitution de l'insula de la place semi-circulaire. 
noirätre. Mans les sondages 26 et 27 ont été mises au jour deux énormes pierres cubiques d'un volume d'un $\mathrm{m}^{3}$ environ. Elles ne sont pas alignées mais elles semblent avoir été installées, lors du dernier état de la voie sur son flanc oriental' ${ }^{16}$.

La rue est-ouest (située sous la rue de l'Abbé-(iellée) présente les mèmes recharges et les mêmes niveaux ; reconnue sur près de 5) m dr long, clle est beaucoup moins large que la précédente. soit environ $10 \mathrm{~m}$. La mesure a pu en ètre prise au niveau du sondage 12, la coupe en celui du 24 . In double alignement de dés de pierres de $60 \times 60$ espacées de $2 \mathrm{~m}$ a 2,30 m annonce les vestiges d'un portique ou plutot d'une galerie. Ies fondations d'un trottoir surélevé ont été apercues en 1975) (sondage 23), le long du coté nord de la rur.

Si nous mesurons la distance qui sépare l'axe de cette voie est-ouest de celui de la rue Saint-P'ierre, nous obtenons te chiffre de $102 \mathrm{~m}$, soit environ 30:) pieds romains ${ }^{17}$. Lin reportant la même distance sur un plan général du secteur, nous aboutissons là où devait passer une autre voie parallèle de ceile-ci, nous avons deux tracés, l'un au sud à proximité de l'ancienne place du théatre ${ }^{18}$, plus exactement te long de la rue Jean-last (emplacement du Foyer des Jeunes Travailleuses), l'autre au nord de la cathédrale, a l'angle de la rue SainteMarguerite et de la rue Nicolas-Pastour ${ }^{19}$. Line autre découverte en juillet 1968 celle-ci, enrichit également notre connaissance de la voirie antique de Beaurais. L'ouverture d'une profonde tranchée destinée à recevoir des càbles téléphoniques fut loccasion de la découverte de 3 couches de silex sous l'empierrement médiéval de la rue Gambetta, en face de l'actuel immeuble des Postes ot Télécommunirations ${ }^{20}$. Ces arguments ainsi réunis, il nous semble permis de localiser nos décourertes (fig. 3) 3$)^{21}$ all sein d'une insula de 102 m de còté.

J'autre part, si nous prolongeons la voie qui traverse notre (hantier du sud au nord, nous relevons deux traces attestés vers le nord dans l'axe de la grande voie romaine qui ronduit, vers Amiens ${ }^{22}$. La largeur de cette voie, son allure générale sont les indices sérieux en faveur d'un cordo tandis que laxe du decumanus aurait été repris par le tracé de la rue Saint-Pierre.

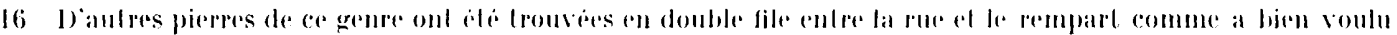
nous lo sigmaler J. Kloin-Bardagri. I quoi pourairnl correspondere ces bloces ?

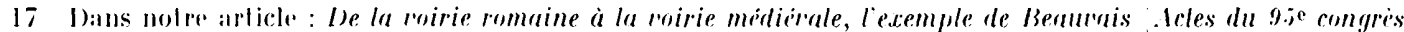

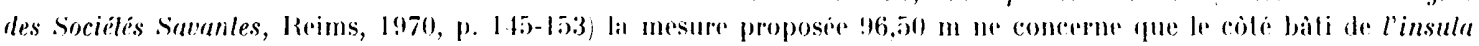
fit non que la mesure intreaxe.

18 Eille a bli fonillé par Piorre I)envis, lin conp de sonde..., op. cil., ogam, XV, 1963, p. $19-6.1$.

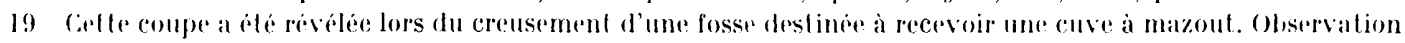

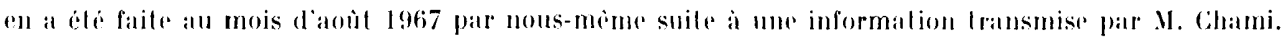

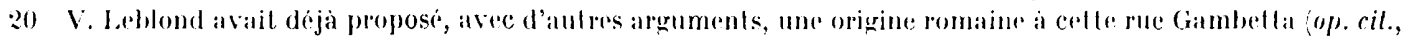
1). $1: 3$

21 A comparer avec les insulae de Silchester; cif. Gerges a. Boox, silchester: The roman town of calleva, I.ondres, 1971, 2 e ed. Il pent paraîle gratuit pour l’instant de reporter celle maille carré de 102 m sur tout le territoire de Beatuais chant donne l'absence de preuves foumies par des coupes de chaussés. comme ce fut le cas récemment a Amiens. Mais le report amine cependant des coincidences troublantes a ansi si nous admettons d'est en ourst un

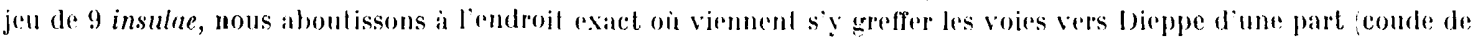

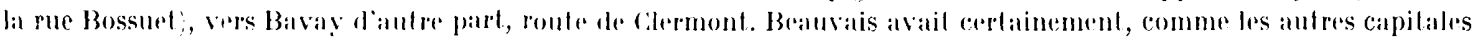

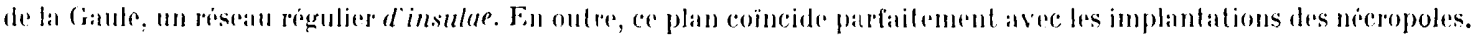

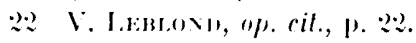




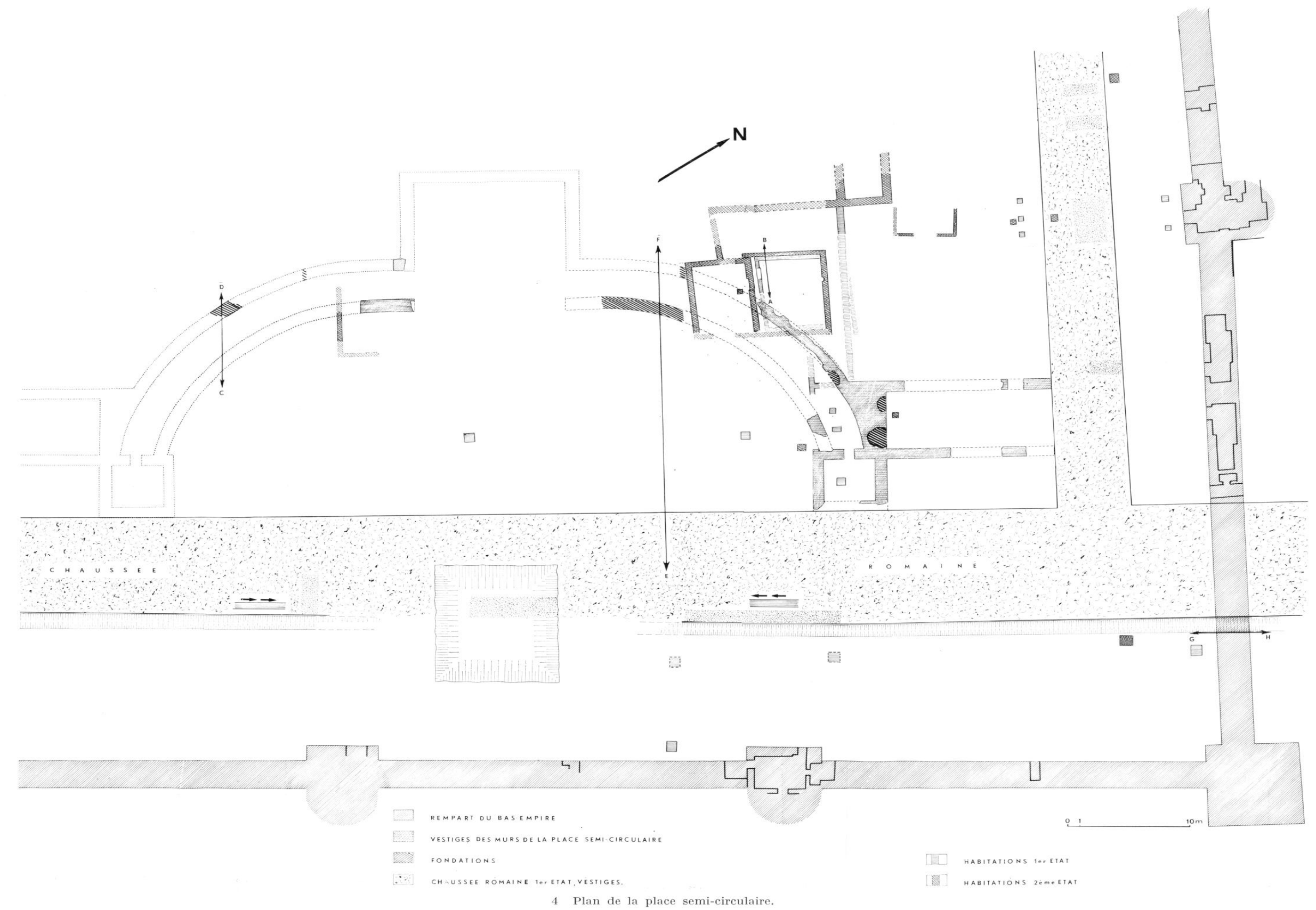




\section{L'occupalion du sol.}

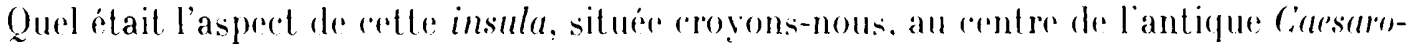
magus? Avant lédification de la grande place demi-circulaire, plusirurs habitations privérs avec cours, jardins et galeries y orcupairnt les lieux. Deux etats dre construction au moins ont été décelés. En plusieurs endroits (sondages 3, 25, 27) nous avons poussé l'exploration jusqu au sol vierere. La cote I.(i.X. (62,5) ni correspond an niveau de la nappe phréatique.

Au niveau 64, apparaît un lit de crair bien tassée, épais deune vingtaine de cm, de quarante par endroits. läst ce lit de craie qui recut les fondations du rempart de la ville au Bas-Empire ${ }^{23}$. Dans le sondage 26 . nous arons, en 1966, pu etudier les couches de lerres. qui s'étendent sous cette chape de craie. Deux niveaux sont bien distincts : le plus profond

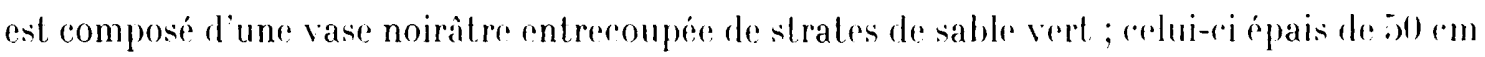
environ sous la rraie contient des débris divers : fragments de tessons, hélas, peu identifiables, de nombreux lébris de bois carbonisés, des petits moreraux de craie. Ce niveau a été reconnu, nous larons dit, sous la fondation du rempart dans langle nord-est du rempart, dans le sondage 3 (déblaiement d'une poche médiévale; foulle d'un puits médiéval par II. Jean (Gartier en 1968) et dans le sondage ?1. En un seul endroit, dans le sondlage :3, fut remarqué un sol planchéifiéct.

Il semble bien établi, dans les limites de notre insula du moins, que sur des terres boueuses gisait un niveau marqué par la présence humaine, qui n’a pu ni être définie ni datée ${ }^{25}$. Au-dessus de ce niveau, fut étendue une courhe de craje bien tassée. sur celle-ci s'élevèrent les murs des maisons et les colonnades du premier état. Ie celui-ci nous n'avons. qu'un aspect partiel étant donné l'ampleur des destructions occasionnées par les récupérateurs de pierres qui ont agi dans ce secteur du lias-Empire aux temps modernes se $^{26}$ Cependant il est possible de donner un apereu d'ensemble sur les différentes constructions érigées en ("rt endroit27 (fig. 4).

La seule habitation dont il fut possible de bever le plan et de préciser la chronologir: se situe dans la partie nord do la place demi-circulaire fign. 1, no 1). Sa décourerte el sa fouille pendant la rampagne de 1967 furent d'ailleurs à l'origine de mesures destinés a l'oulverture d'une campagne de fouilles plus etendues et surtout a une enonservation in silu des vestiges dans une reyple arehéologique sous la Cialerie de la Tapisserie. La piece mesure.

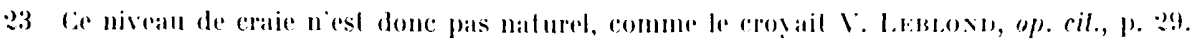

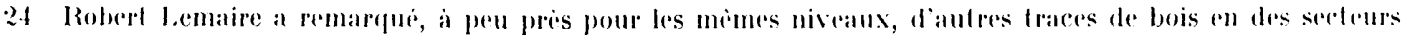

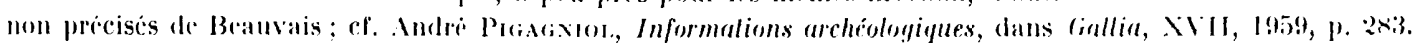

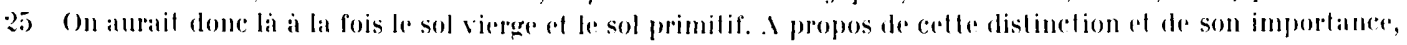
voir la communication, à propos d'Alba Fucens, de J. Mretsis, Thimes de recherches sur les rilles anliques docridenl.

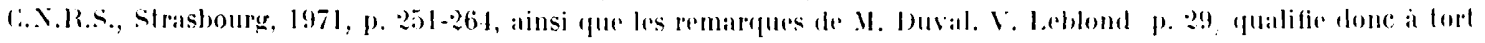
ce "cran" de sol primitif.

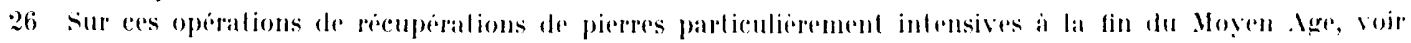

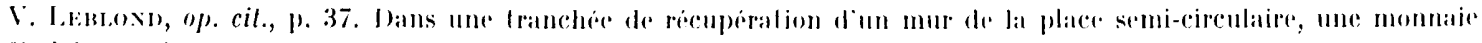

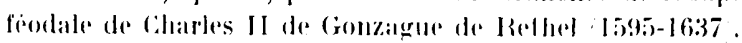

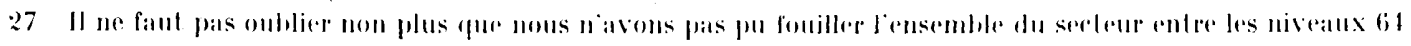

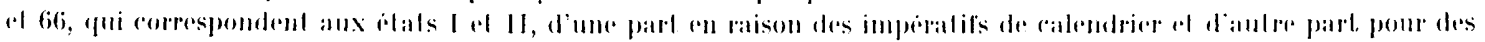

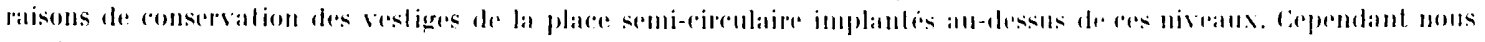

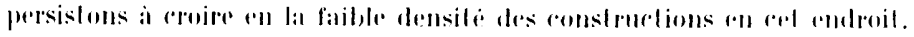




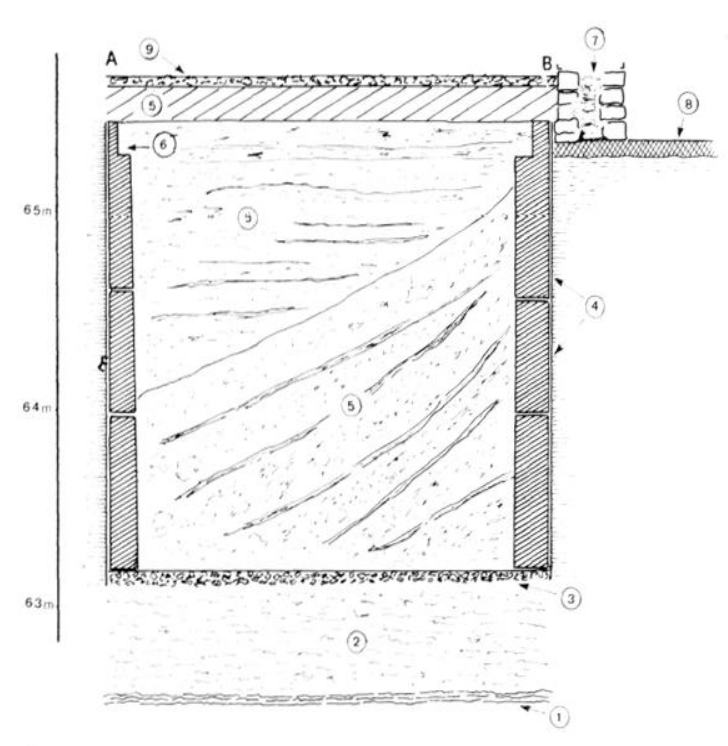

coupe en $A B$ et les deux états de la cave de la maison

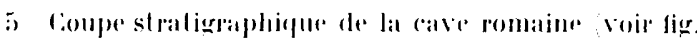
1, 13, : 1, nivean actuel de la nappe angufere; : terre fangense ; 3, sol de la cave an crate dammere; 1, mur rol

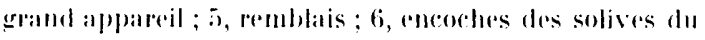

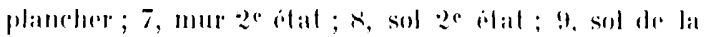
maison $:$ aral.

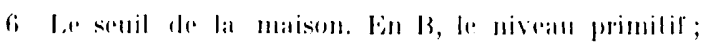
ill le serond inlal.

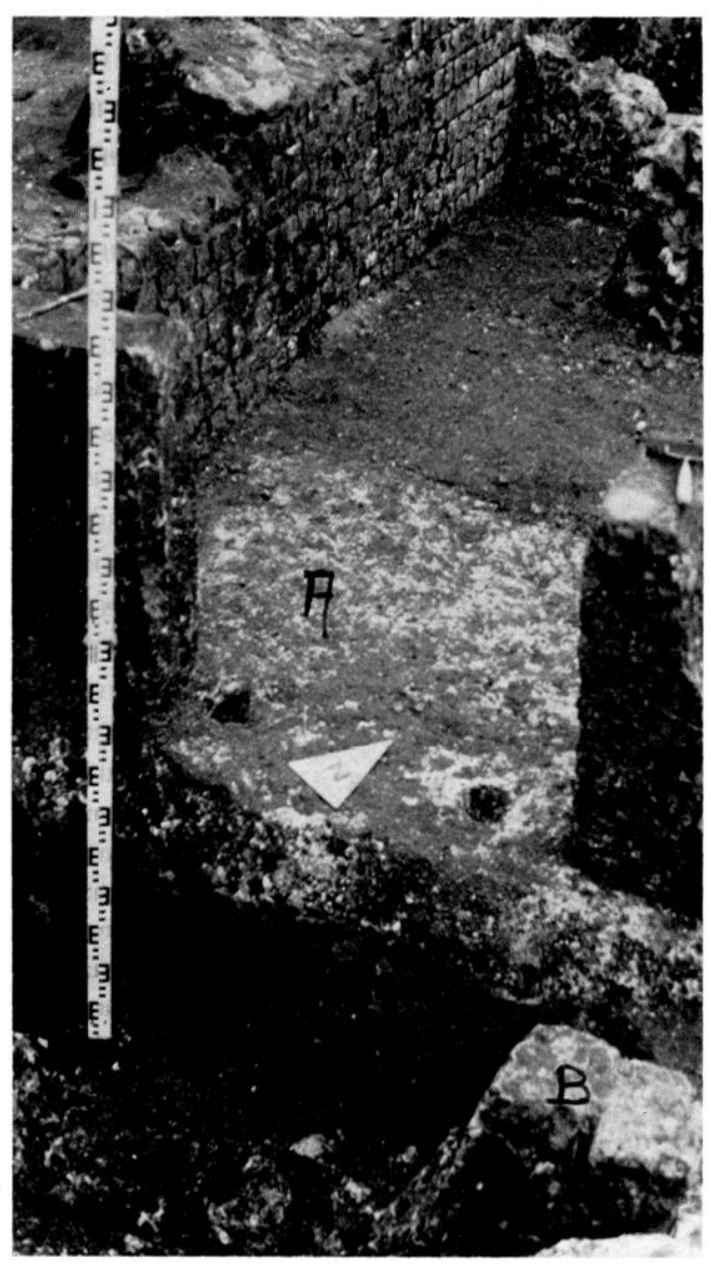

dans son premier état, 5,70 $\mathrm{m}$ sur $6 \mathrm{~m}$ de long, avec une cave large de $2,4 \mathrm{~m}$ aménagée sur son flane occidental. Le senil d'entrée, large de $1.10 \mathrm{~m}$ porte deux trous de 8 cm de diamètre. L'omplacement de ces trous, dans lesquels devait itre fichée la lige de fer destinée a maintenir le montant du bati en bois, permet de supposer une porte de 80 c'm c'nviron de larges (fig. i) et 6$)^{28}$.

Cette piece du premier etat, rappelons-le, etait installese sur une cave batie en grand appareil irregulier identique a colui des caves romaines de la forel de Pierrefonds²9. La cave de Braurais est construile a l'aide de grandes pieres carrées, de près d'un metre pour 25) con de large et posées a joint vif. L'une delles a elé percée a fin d'y aménager un pel it soupirail. leeux empreintes de solives ont éte remarquées sous te flane ouest, solives qui

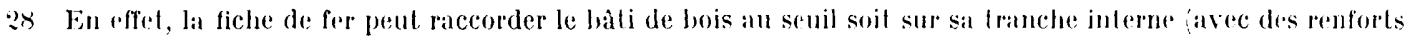

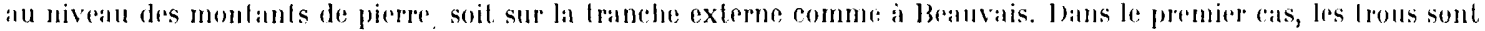

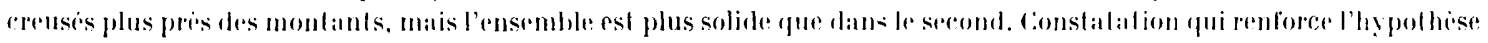

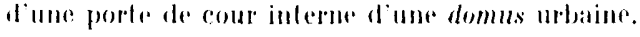

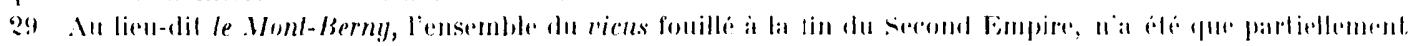

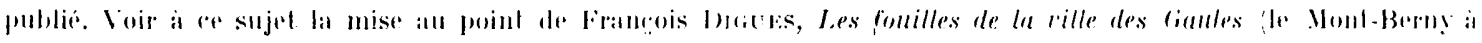

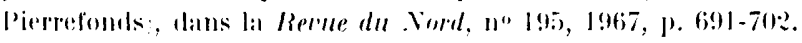




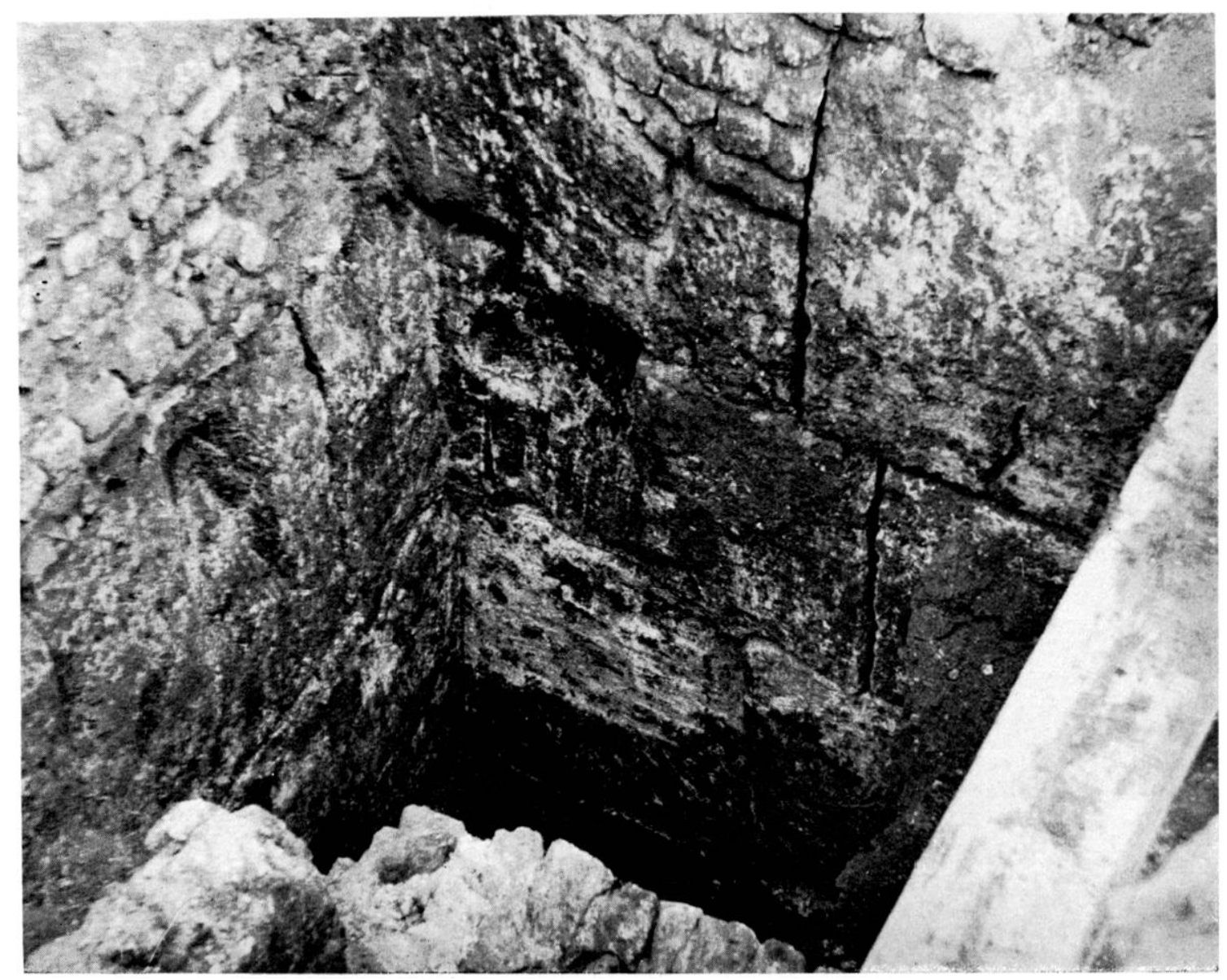

7 Vie de l'appareil de la cave romaine.

devaient supporter un plancher (fig. 7). C"est à cette pièce que devaient se rattacher plusieurs murs d'une grande maison dont la façade sur rue devait itre agrémentée d'une colonnade. Le mème niveau a été repéré plus au sud au niveau du mur est-ouest, large de $40 \mathrm{~cm}$ (fig. 8) et dans le sondage 21 par une base de colonne (Inv. II, 10) $)^{30}$. A ce niveau, gisait un petit dépotoir empli de fragments d'enduits peints de couleur bleue en compagnie de céramique typique du $\mathrm{I}^{\text {er }}$ siècle ${ }^{31}$. Un autre dépotoir au niveau de la rue est-ouest (sondage 24) a fourni un matériel de mème époque.

Le passage au deuxième état, bien visible au niveau 6.) $m$ I.(i.X. a bien été repéré au niveau de la pièce sur cave el de ses annexes (fig. 7). Les raisons n'en sont pas connues (il n'y a pas de traces d’incendies bien évidentes) mais nous savons que toutes les villes romaines de la Gallia Belgica ont connu plusieurs phases de développement. La cave précédemment, étudiée est à présent romblée $e^{32}$, et un niveau de craie tassée, épais de 10) ('m, est épandu

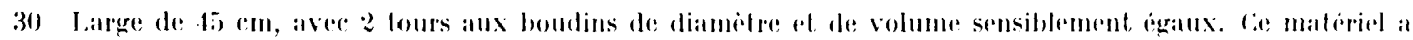

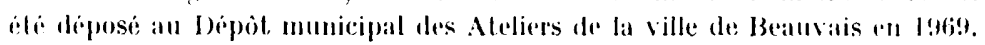

31 lin maleriel sera publie dans une prochaine étude.

3: Comme nous l'avons dit, le remblai romain de la care a ite repere pour laissere place i une fosse d'aisance

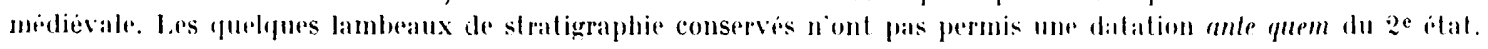




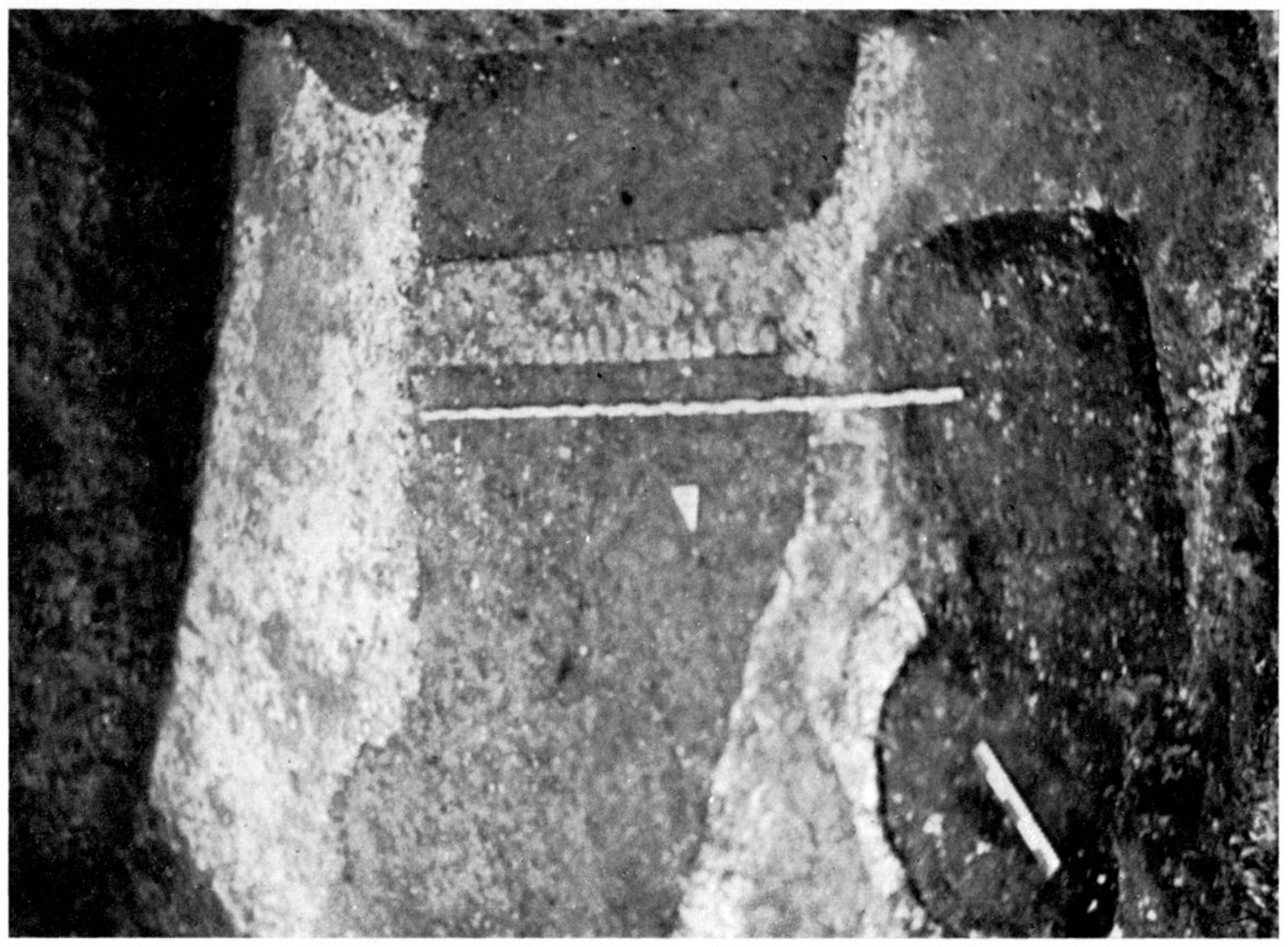

\& Vur sur les massifs de fondation de la galerie de la place semi-circulaire. lin mur d'un etat anterieur a été recoupé, un puils mediéval y a ble perce ainsi ru'une fosse rectangulaire (le nord-est à gauche

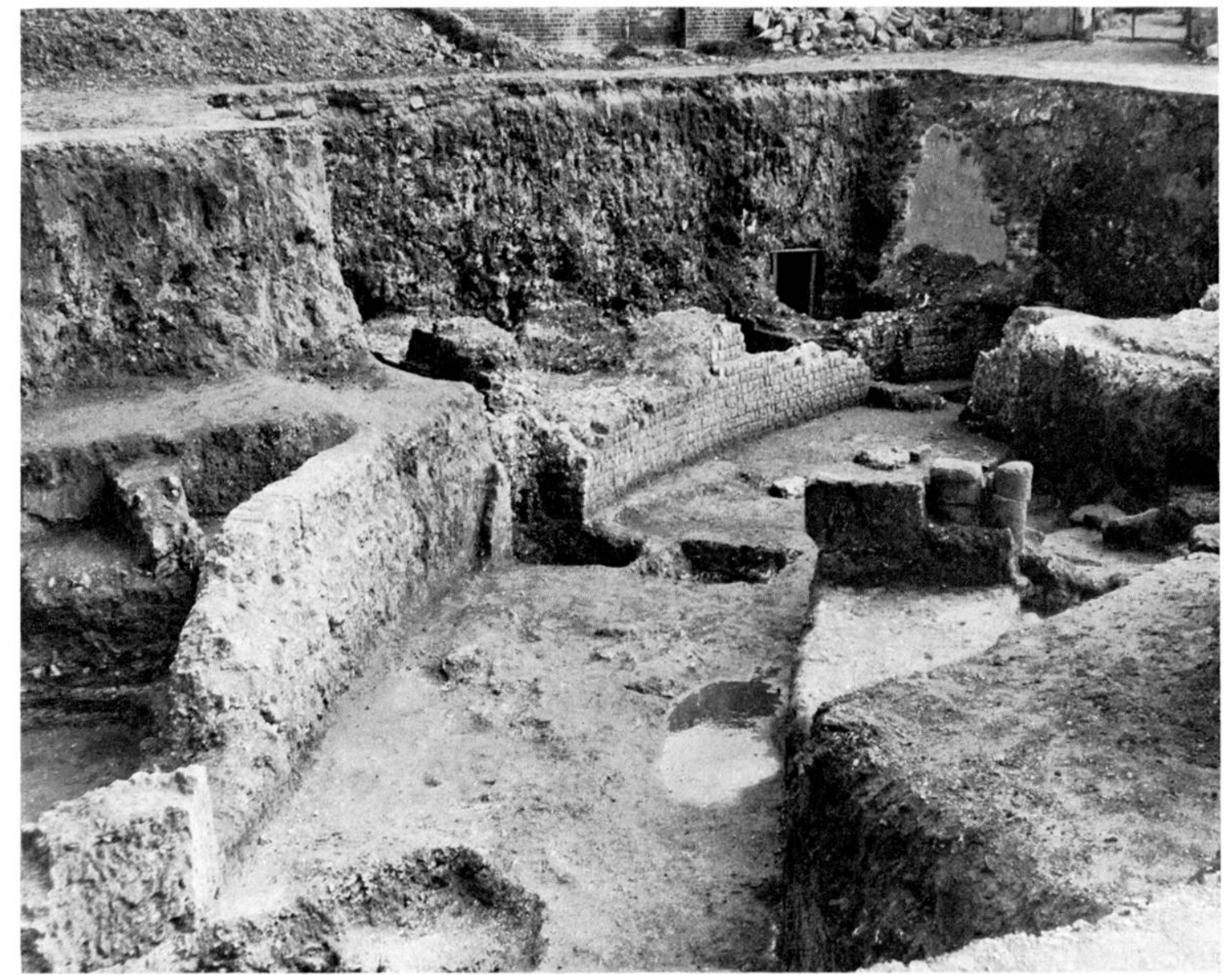

9 Vue sur les vestiges du mur externe de la place semi-circulaire. 


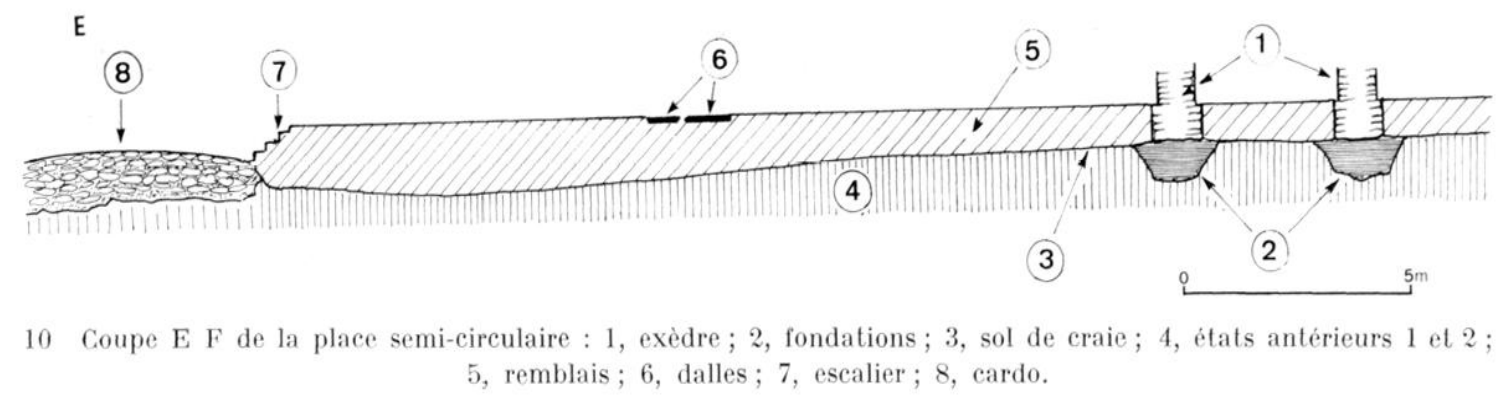

sur lequel on assoit un noureau seuil, identique au premier foir fig. 6). Le mur oceidental est doublé par un mur en petit appareil. I) facon générale dans tout le chantier, le nivean général est rehaussé de $10 \mathrm{~cm}$ à un mètre. Le niveau des deux rues est rechargé dans less mèmes conditions. J)ans le sondage 1, a été mis au jour un mur qui appartient à ce deruxième état. Le report sur plan (fig. 1) scmble apporter la preuve d'un remaniement du plan de la domus. I e matériel ceramique, riche an poteries grises a col tronconique est typique de la fin du $\mathrm{II}^{\mathrm{e}}$ sièrle. Plusieurs menles intactes ou en fragments ont également ete découvertes ainsi que des objets de bronze. Les enduits perints sont relativement rares et sans décor particulier. Il convient de noter également une différence dans l'appareil des murs, a la fois dans lépaisseur des joints et la composition des mortiers, par rapport au premier état. La documentation partielle, rappelons-le, fournie par la fouille de cette insula est assez banafe par rapport à l'histoire des grandes villes de la Ciaule : un départ assez lent de l'urbanisation, un plan assez làche des appartements de grandes maisons urbaines, morlifié au moins deux fois aux $\mathrm{I}^{\mathrm{er}}$ et $\mathrm{II}^{\mathrm{e}}$ siècles avec un rehaussement systématicque du site ${ }^{33}$.

Moins banale fut l'implantation d'une très grande place semi-circulaire dont la consIruction occupe toute la surface de l'insula, ensemble unique sur le territoire de toute la (iallia Belgiea (fig. 1).

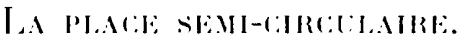

Malgre les extrèmes ravages provoqués par les recopienateurs de piereres de pierrailles. le crensement des caves médievales, le plan d'une place semi-circulaire a pu etre dresé arec cependant une incertitude en ce qui concerne la partie centrale de la courbe qui na pu ctre fouillée (fig. 9).

Ine place monumentale a done ste élifiese a plan semi-circulaire, alene i chaque

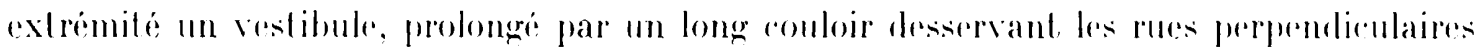
au cardo maximus.

Parfaitement inscrit dans le radre de linsula. le plan n'est pas sans évoquer des réalisations en exerdre rheres an repertoire de larehitecture romaine ${ }^{34}$. Equidistants de

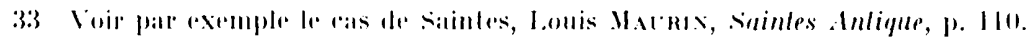

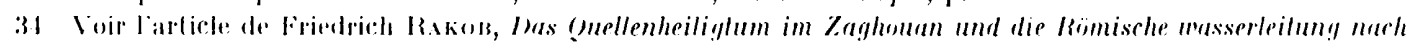

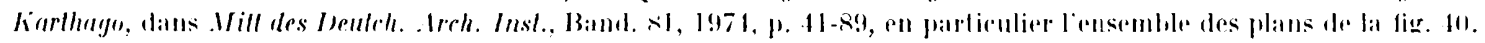




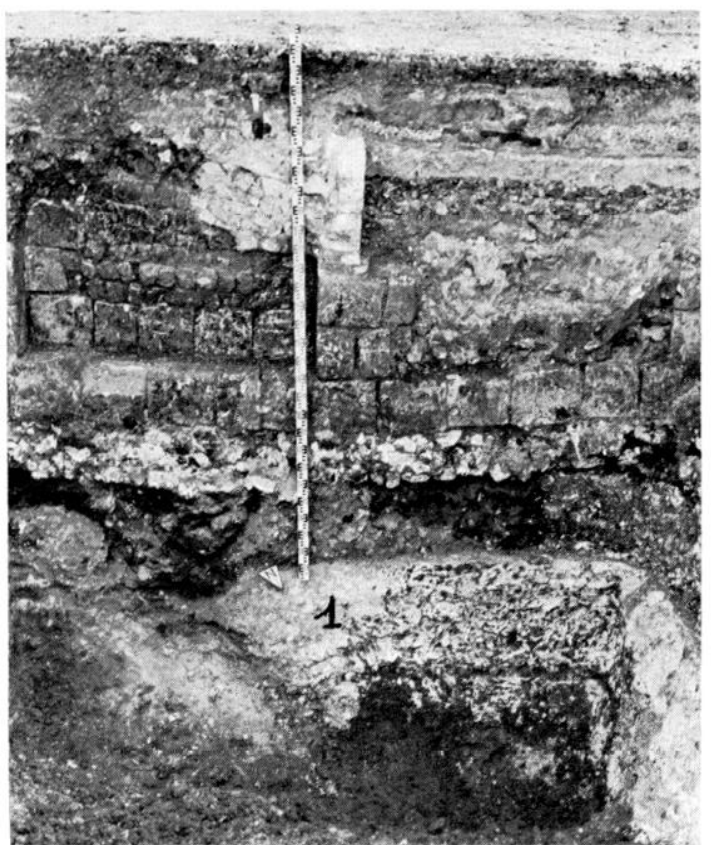

11 Dans le sondage 21, la photo fait apparaitre en 1 Ir massif de fondations de lat branche sud de la galerie Ir la place semi-circulatire. Complelement délnarrassée de sa construction, elle a servi d'appoi a des murs d'iporgues postirientes. de bois; 2 , terres moires ; 3, sable; 1 , terees el charbons de hois ; 5, silex lies au mortier; 6 , silex, tuiles, braises ; $\bar{i}$ teres, silex, pierres; $\times$, remblai.
$1: 3$ Coupe des drains du cardo: 1 , joinl en fer, debris
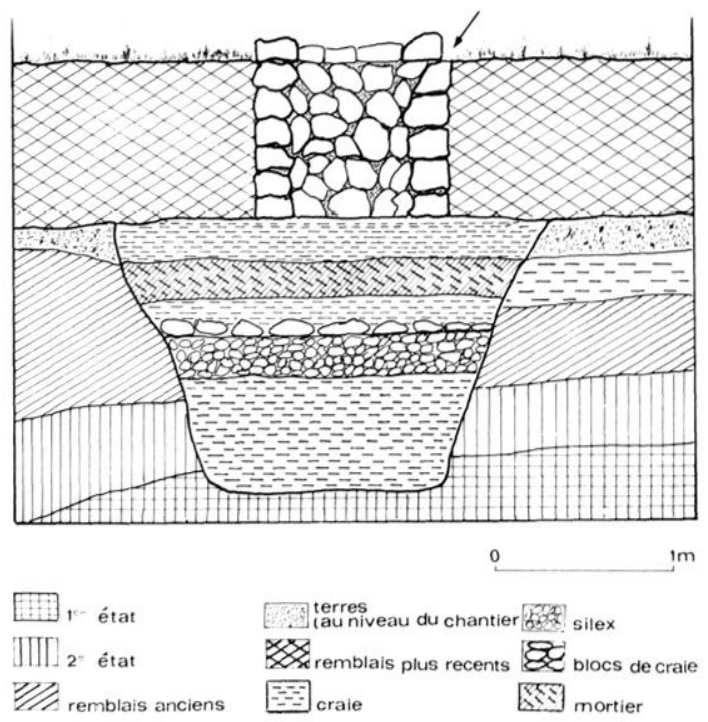

1: lilude slmatiglaphiefue des fondalions des murs de la place sobre igalement la fig. lo,.

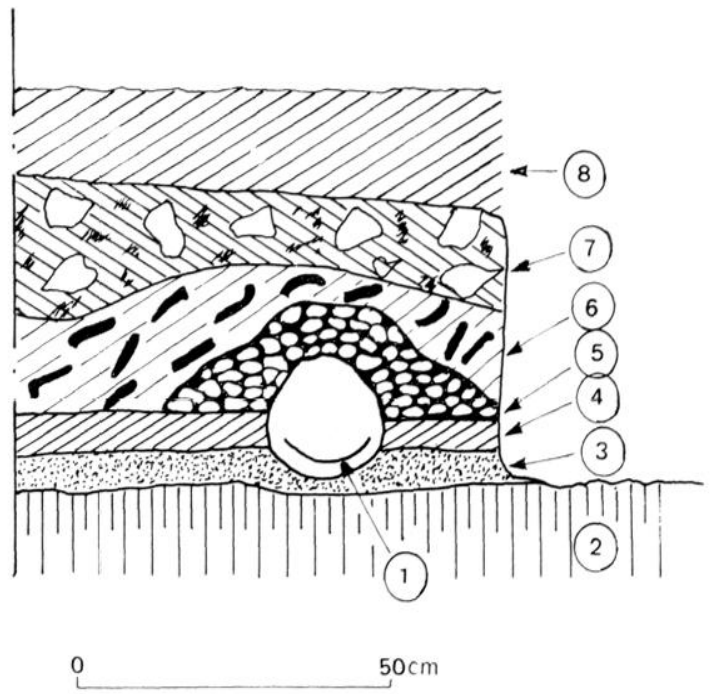

j2,50 m, deux pièces au plan quasi rarré sont reliées par deux murs parallèles tracés selon une demi-rourbe, rompue en son milieu par une grande construction rectangulaire ou également semi-circulaire. Des drux éléments carrés sur lesquels s'appuic ainsi le grand exèdre divergent de part et d'autre deux grands murs paralleles équidistants de 5,30 $\mathrm{m}$. Ceux-ri ont ite construits en mème temps que chaque pièce carrée à laquelle ils sont raccordés au sein d'un mème massif. La présence des talons internes et externes (fig. 10) de quelques ééments de dallage, permettent de reconstituer les niveaux enfermés à l'intérieur de ces murs. Iess talons sont situés au niveau 66,00 - niveau auquel correspond la masse de remblais mi-argileux, mi-craycux sur lequel devait reposer un dallage (3 éléments seulement en furent retrouvés isolément dans le sondage 8 en septembre 1969). Les mèmes niveaux 


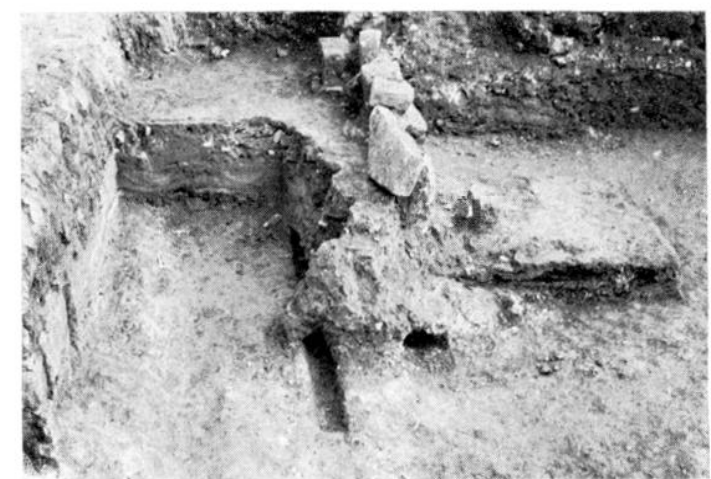

14 Vue des empreintes des drains.

15) Coupe stratiographique du massif de fomdalion du rempart: 1, torees fangreuses; : lerees noires, sable, debris divers, lessons, hois ; 3, sol du Ilaul-Empire;

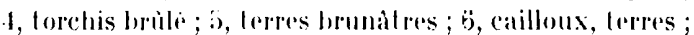
7 , drbris crayeux lres fins; $x$, dechels de pieress calcaires blanches ; 9, chainages ; 10, remblais recents ; a, b, c, d, sols surecessifs du Bas-Fimpire ad du Moyen . Ige.

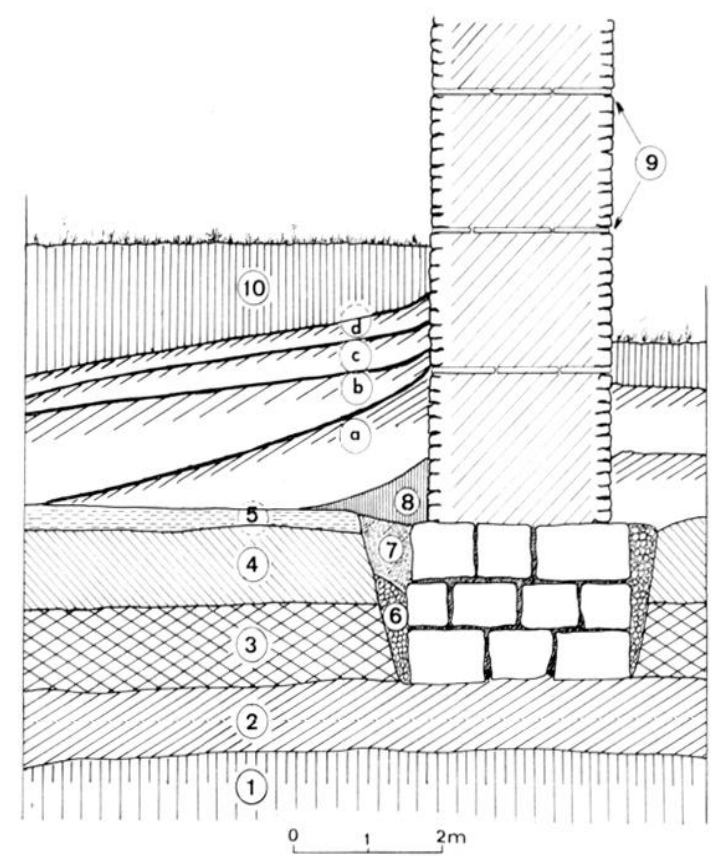

étalons ont été également retrouvés sur le mur sud de l'exèdre (juillet 1969, sondage 10). Si nous pouvons assez aisément reconstituer le plan de la partie nord de l'exèdre (sondages 1 , $2,3,4,12,22)$ la tentative au sud s'est avérée beaucoup plus difficile (sondages $7,9,10$, 11, 21). En 11, nous espérions, et ceci en septembre 1968, retrouver un ensemble symétrique: a celui du nord, par simple déduction de symétrie.

l'ouverture des sondages 7. 9, 10, en juillet 1969, devait nous révéler te tracé de la branche sud de l'exedre, de telle sorte qu iil fallait conclure à une rupture du trace semicirculaire de l'exidre en son rentre par la construction d'un ensemble a plan carré ou courbe. Mais la présence de grandes caves médiévales devait empercher toute exploration lans ce secteur. La confrontation ave un relevé stratigraphique effectué en juin 1967 (sondage 21) allait emporter lindice décisif en faveur du tracé de la branche méridionale: de l'exishe, parfaitement symétrique a partie septentrionale (fig. 4, 8, 9, 10, 11, 12).

En trois endroits nous fut donnée l'occasion d'examiner les fondations des murs courbes et perpendiculaires dans la partie nord de l'exèdre. Sur le site détruit des habitations urbaines, une épaisse couche de remblais fut épandue, a travers furent percées deux larges tranchées dans lescluelles furent montées les fondations des murs de l'exìdre (fig. 12). Leurs dimensions, le soin apporté a leur construction sont remarquables. Ces murs soutenaient-ils une colonnade ou un alignement de pilastres ou un mur évidé d'arches régulières ? L'absence d’élévation des restiges nous empèche d'en dire davantage. Mais le grand nombre de colonnes retrouvés dans le chantier, au niveau de la couche de la démolition antérieure a lédification du rempart du Bas-Empire, nous incite à supposer la présence d'une colonnade el done d'un portique, d'autant plus que dans le sondage 3, fut découverte une colonne gisant sur le sol de la place semi-rirrulaire. 
I rel consemble doit etre associe un riamenagement de la rue sur layuelle il s'appuire. Les sondages 17, 19, 20) (de juillet 1968) ont mis en evidence en mème temps deux ronduites en bois installées tout le long du cardo. Ces deux conduites devaient déverser leurs eaux, à en juger par leur pendage respectif, vers un bassin à plan rectangulaire, creusé juste dans laxe central de la place semi-circulaire ${ }^{35}$ (fig. 13).

I)'où provenait l'eau ainsi recueillie au niveau de ce bassin en bois ? Eaux de pluies provenant du monument central de l'abside ou de la rue elle-mème ? Eaux venant du soussol très humide et amenées par un jeu de conduites dans un bassin construit aménagé dans laxe médian de la place semi-circulaire? Pendant un certain temps, cette hypothèse a retenu tous nos suffrage's; mais les sondages profonds $(8,7,9,10,14)$ n'ont pas livré la moindre trace de conduites d'eau drainant le sol gorgé d'eau, dans le sol de la nappe phréatique, s'écoulant d'ouest en est (voir fig. 2). Malgré les incertitudes dues aux bouleversements du sous-sol dis le Bas-limpire, il est possible néanmoins de reconstituer la masse imposante de cette place semi-circulaire devant laquelle gisait une piece d'eau alimentée par des conduites en bois enterrées dans le sol de la rue principale (fig. 14). Quelle que füt sa fonction, cet ensemble derait, par sa position dans la ville antique dominer l'horizon des maisons ef des édifices civils de l'antique Ciaesaromagus, et constituer peut-ìtre l'élément majeur l'un forum remanié à lépoque des sévères.

Cette transformation urbaine est à inscrire, croyons-nous, par son ampleur et sa volonté délibéréc de prestige dans le grand mourement de transformation urbaine de la fin du $\mathrm{I}^{\mathrm{e}}$ sièrle, dernier érlat de la P'ax Romana avant les catastrophes du sierde suivant.

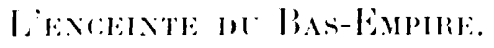

Cirice aux travaux du lor Leblond, puis reux de R. Lemaire, nous connaissons bien le tracé de l'enceinte ${ }^{36}$. Tongue de $1370 \mathrm{~m}$, elle protégeait une zone d'environ 10 ha 70 a. Aux tours carrés des angles sucededaient comme a senlis, des tours semi-circulaires régulièrement espacées. La fouille de 1969) a mis au jour une tour inconnue jusquà cette date. II. Robert Lemaire en avait soupconné l'existence d'après ses calculs d'intervalles depuis la tour d'angle at la porte d'entrée du caslrum, encore bien visible sur les plans du xvire siècle ${ }^{37}$. si la face extra-muros a été détruite, le saillant intra-muros a pu étre observé d mesuré dans de bonnes conditions (largeur $5.90 \mathrm{~m}$, saillant 1,35 m). Ainsi, près de vingt

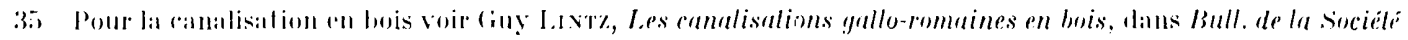

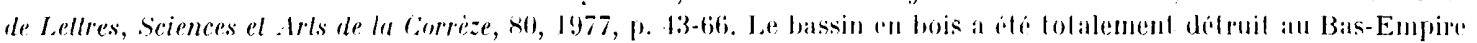
far une profonde rxcavation. Ia mise al jour d un angle preserve a premis sa reconstilution partielle. Il convient. de remarquer igatement sa position dans laxe majeur de l'écoulement de la nappe phreatique d'ouest en est fige I ). dree davantage d'arouments monumentaux (conduites, hassin all phan mienx défini), on aurait pu evoquer une arrande fontaine on une nymphe de type de Kaghouan.

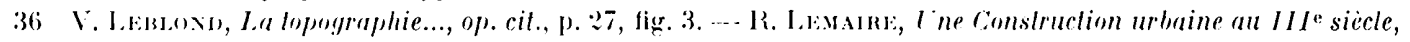

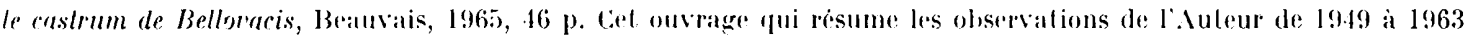
est ignoré par Stephen Johnson dans sa recension des Villes fortifiess de la Gaule Belgrique !Brilrunnia, vol. IV', 1973, l. : $: 10-9.23$, fig. $1 ;.$

37 R. I.amarre, Lne Construclion urbaine..., p. 25, "notre hypolhese est que sur la dislance de 101 m (qui sipare

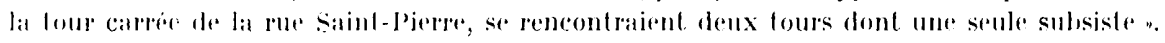


ans après les recherches de MII. F. Vasselle et R. Lemaire un nouvel élément permet une reconstitution du plan de lenceinte du Bas-Empire ${ }^{38}$.

Différents sondages de contròles ont livré plusieurs renseignements intéressant le mode de construction du rempart et de son élévation. En premier lieu. il apparait que la tranchée we fondation descend partout jusquau niveau de craie contemporain du premier état de la ville antique ${ }^{39}$. Cette tranchée est ouverto à partir d'un niveau de remblais composés de restiges les plus divers : lébris de torchis, bois calcinés, moellons brisés, terres grises et noires. Ce niveau de remblais, épais de près diun mètre (fig. 15) a recouvert uniformément le site au nord de la rue est-ourst et à l'est de la rue nord-sud. I)ans la tranchée ainsi ouverte large de plus de $3 \mathrm{~m}$ sont descendus les bloes appareillés qui, disposés en triple lit, constituent la fondation de la muraille. Celle-ci large de $2,50 \mathrm{~m}$ est constituée d'un petit appareil régulièrement parcouru de lit de grands carreaux de terres cuites. Plusieurs blocs sculptés ont èté retrouvés en particulier dans la cave creusée à mème le massif de fondation sous la tour Saint-Ililaire ${ }^{40}$ ( fig. 16). Si certaines appartiennent au répertoire des stèles funéraires et doivent provenir des nécropoles des marges de la ville, l'autres sont issues de grands monuments (entablement, corniches, colonnes ciselées, etc.). Or. sur le chantier 1 nous avons fait dégager un alignement de blocs de pierres régulièrement disposés en arc de cercle depuis la partie centrale de la place monumentale. Ceet alignement de pierres régulièrement disposées en double file gisait sur la couche d’incendie du Bas-Empire et venait juste à l'encontre de la rue romaine nord-sud (fig. 17). Par leur volume et leurs dimensions, ces pierres sont exactement semblables à celles que nous trouvons dans la fondation du rempart. Comme nous le suggérons à l'aide de la figure 18, nous pensons voir les vestiges du chantier de démontage organisé systématiquement à partir d'un grand monument installé dans l'axe de la place semi-circulaire, approximativement à l'emplacement du chevet de la cathédrale ${ }^{41}$. Les pierres auraient été amenées ainsi jusque la rue pour être réparties de l'autre còté sur les chantiers de taille répartis çà et là sur les terres avant d'être descendues dans les chantiers de construction. La tranchée de fondation mesure $2,50 \mathrm{~m}$ de profondeur pour autant de largeur. Les pierres du massif de fondations, disposées en trois lits réguliers mesurent de $0,90 \mathrm{~m}$ à $1,10 \mathrm{~m}$ de long, 60 à 50 con de large pour autant d'épaisseur. Ces dimensions sont précisément celles des pierres disposées en are de cercle au nord de la place monumentale, aurions-nous là des vestiges d'un ensemble non utilisé de pierres de fondations? Des reconnaissances dans d'autres secteurs du caslrum seraient souhaitables pour étayer cette hypothèse. Les pierres de fondations disposées à joints vifs étaient néanmoins reliées par des scellements en double queue-d'aronde. Nous avons pu le vérifier au niveau d'une grande cave médiévale (sondage :26) située au contact de la tour d'angle du castrum.

Les sept pierres du lit supérieur de la fondation visibles par l'arrachement du parement interne du rempart provoqué par le creusement de la cave sont toutes scellées par des

38 Le plan public dans Britannia est incomplet (loc. cit., fig. 1). Pour la fondation des fours en plan rectangulaire, voir L.FMaIRE, op. cit., p. 8 .

39 Voir note 23, supra.

40 Sculptures déjà repérées an xvite, V. L,ebloxd, op. cit., p. 16. $50 \times 50 \mathrm{~m}$.

$11 \mathrm{Si}$ on tient comple des dimensions de l'insula, voir fig. 3, ce "monument "s'inscrit dans une surface de 


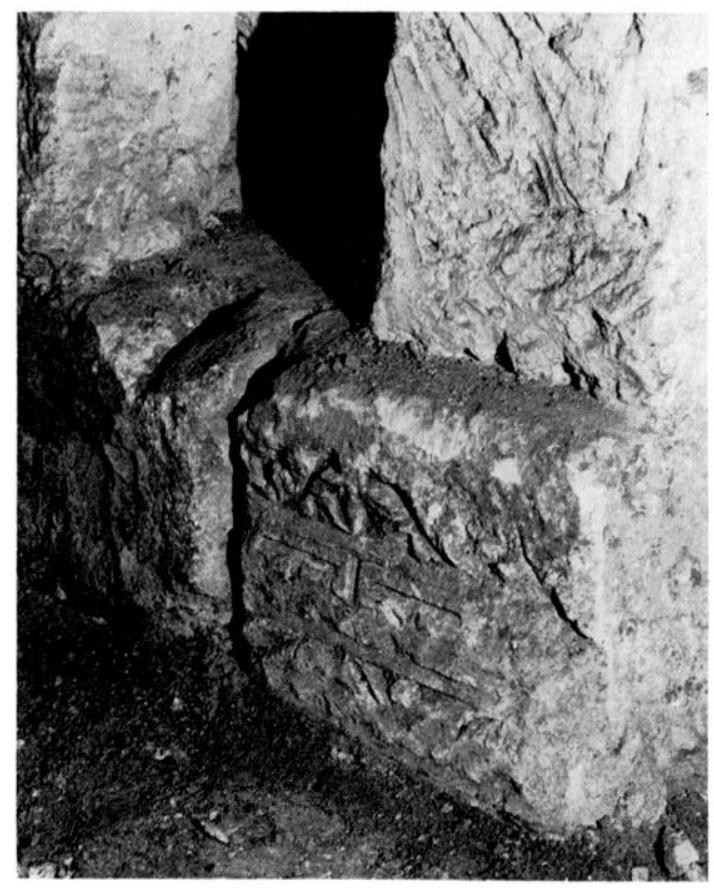

16) Bloc scuptio dans le noyau de fondation du remparit.

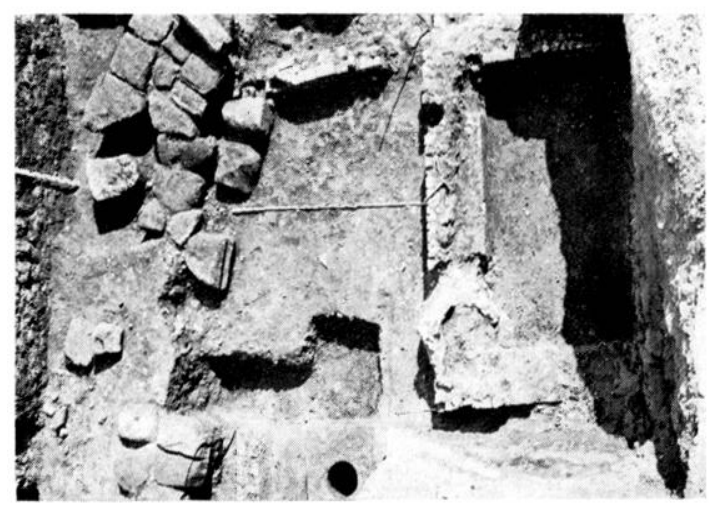

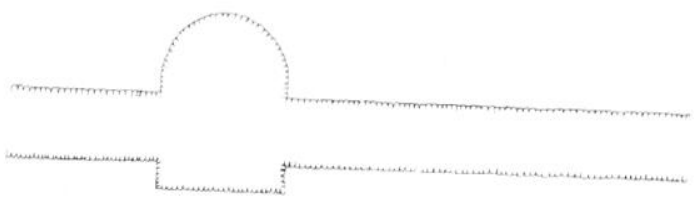

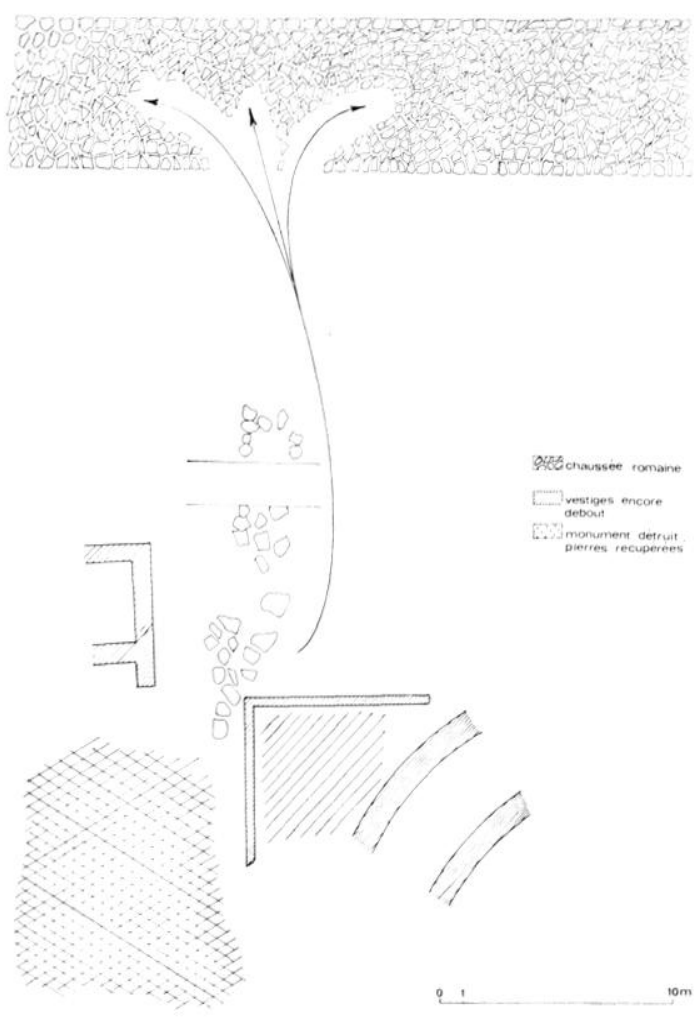

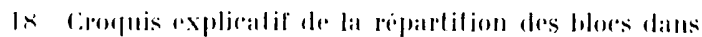
less ruines.

17 . Mignement de hors dissimines dans les ruines momaines.

crampons sans doute en bois. Le mur proprement dit avec ses parements en petits appareils régulierement soulignés de grands carreaux de terres cuites, supportait également des boulins dont les trous sont encorr visibles. L'examen attentif de l'un d'entre eux a permis d'en relever le profil et la masse de mortier prise a l'intérieur de la ravité. Il semble bien que te boulin y a été engagé alors que le mortier était encore frais, avec une forte pression extérieure de haut en bas, ce qui a donné un profil particulier à la cavité du trou de boulin. Nous aurions en cet endroit, mais la chose est peut-itre différente en d'autres points du chantier, la trace d'un échafaudage encastré en bascule. Lin outre, l'examen attentif des niveaux du chantier du remplart, bien visible avec sa masse claire d'eclats de pierres, ne 


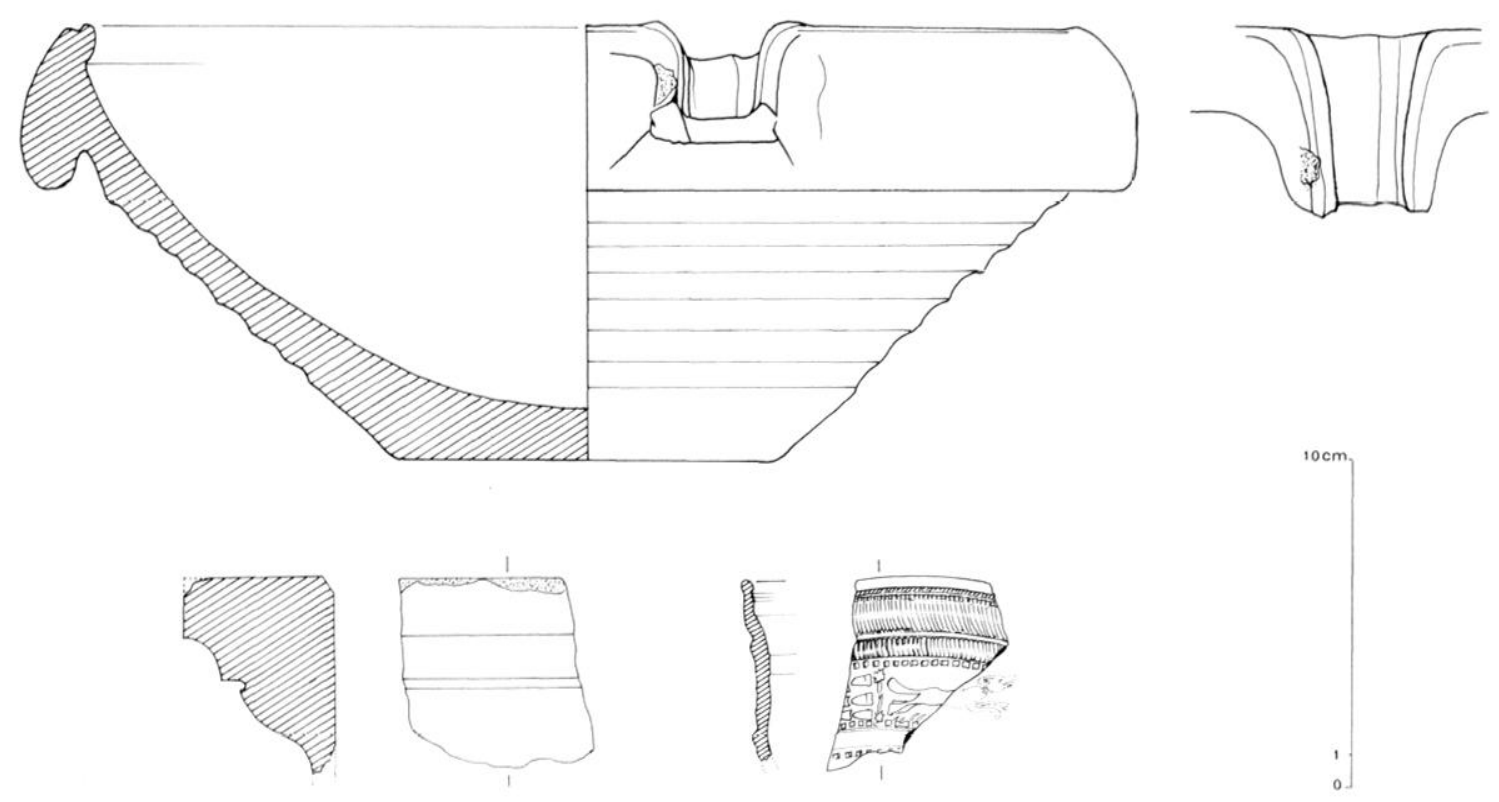

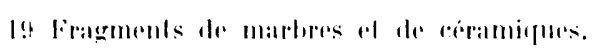

révele aucun trou de fondation de perche d'échafaulage encastré à rang de perches ${ }^{42}$. Dès le chantier terminé, a en croire les renseignements fournis par les relevés stratigraphiques, des remblais ont dù etre ramenés le long du rempart en plusieurs phases, de telle sorte quiune sorte de rampe s'est formée en pente douce de sa face interne jusquen bordure de la

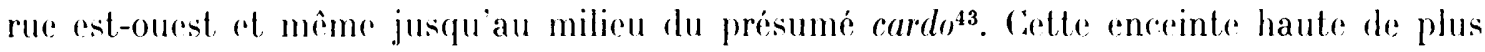
de 5) m all-dessus du niveau antique pour atteindre 7,30 $\mathrm{m}$ au sommet des tours, est datéc par les historiens et les archéologues, de Prohus au plus tot, de I)ioclétien en fonction des monnaies de ces empereurs déecouvertes dans le blocage de la muraille"th.

dipendant nous tenons cette date pour provisoire avec même quelque argument en faveur d'une (hronologie plus hasse. En effet, nous avons décourert dans la masse de pemblais decoupes par la tranchée de fondation du rempart des monnaies frappées au début du I $^{\text {e }}$ s. $^{45}$. A res travaux gigantesques, il faut ajouter egalement ceux qui ont

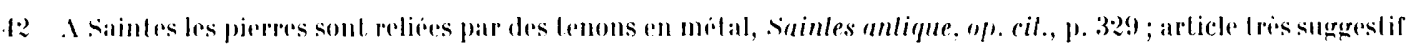

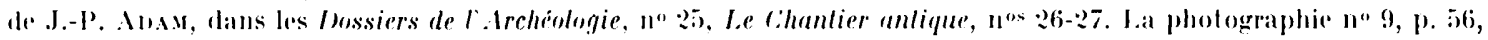

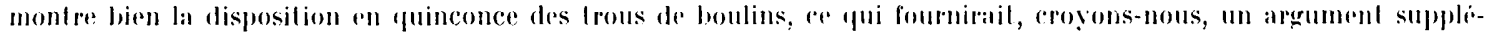

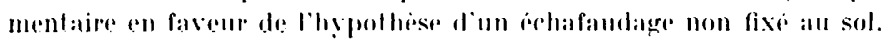

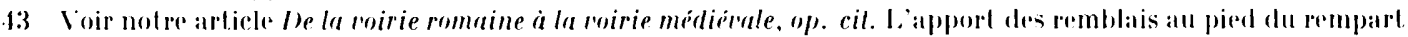

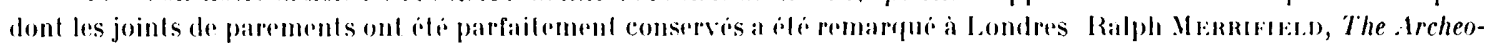

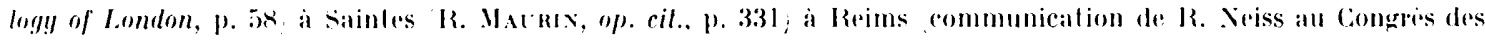

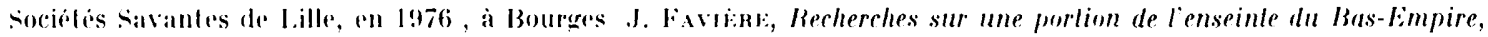

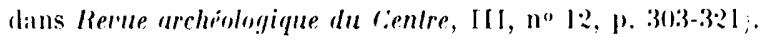

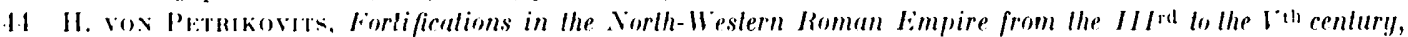

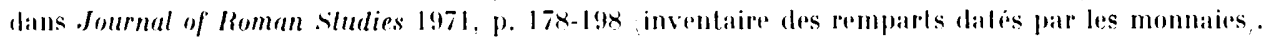

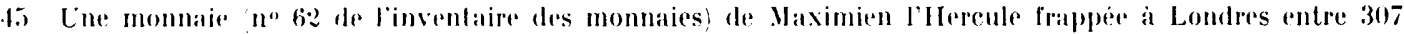

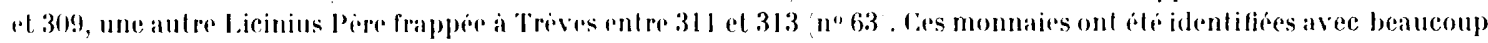
de devonement par Robert Jhenin, decede en 1969, helas! M. Loisel, en 1970, a dresse linventaire des monnaies de la campange: de 1969. 

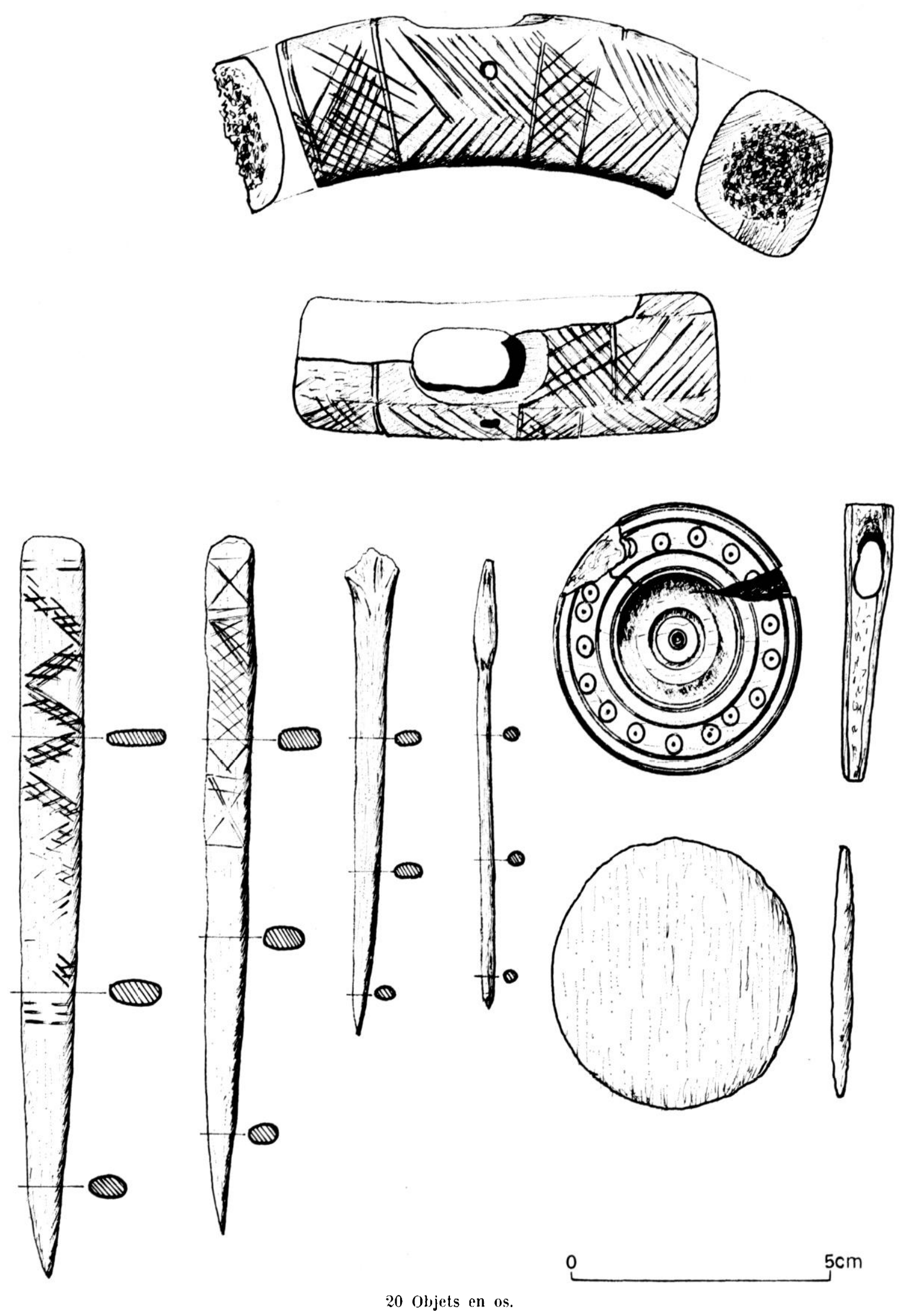


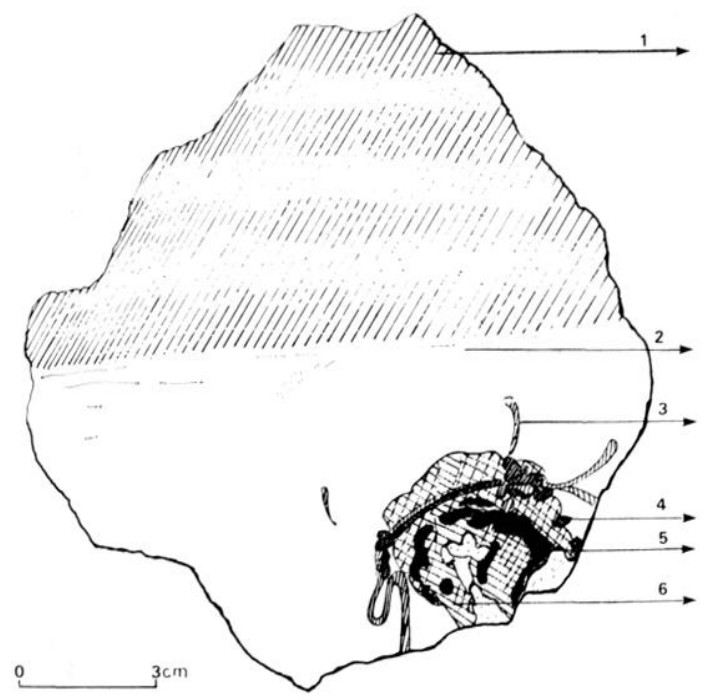

21 Fragment d'rmbit peint. Tute de faune : 1 (2.5.). Y.13/4/4) rouge brun mouchele, 2 (1.0.Y.R./8/1) blanc ; $3,4(5.1 / 5 / 6)$ vert olive (clair $=3$, plus sombre $=1) ;$ 5) (2.5.11/36) rouge sombre ; $6: 7.5 . Y .1 / 7 / 6 / 6)$ rouge pàle.

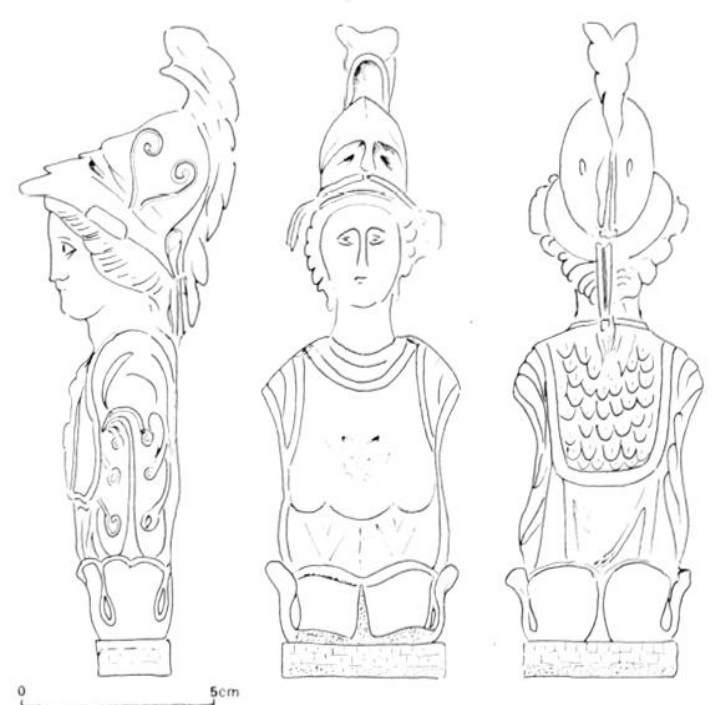

22 Petit bronze : Vénus casquée élement de tripiod ?). (I)essin G. Jelski,.

entrainé le creusement d'un canal de déviation autour de l'enceinte destiné à écouler les eaux d'ouest en est et bloquées par les masses profondes de l'enceinte ${ }^{46}$.

A l'intérieur de la zone ainsi fortifiée, du moins en ce qui concerne notre secteur, hormis des objets caractéristiques du Iv ${ }^{\mathrm{e}}$ siècle $^{47}$, nous n'avons pas découvert de structures datées du $\mathrm{v}^{\mathrm{e}}$ siècle. Dans le sondage 4 , se voit la fondation d'un mur antérieur au Moyen Age (à en juger par le puits de cette époque qui le recoupe) mais nous n'avons pas d'éléments pour le dater avec plus de précision. Ln coin de maison du Haut-Empire a été réoccupè à l'époque mérovingienne avec réfection du sol et pose d'enduits sur le mur.

\section{LI: MATÉRIEI. DÉCOOVVERT.}

Plusicurs éludes seraient nécessaires pour venir à bout d'un énorme matériel amassé pendant quatre ans de fouilles. Nous proposons donc ici un choix parmi les pièces les plus représentatives et intéressantes.

Céramiques. De l'inventaire des signatures sur fonds de sigillée, aucune marque inédite n'a été relevée. Ians la masse de céramiques communes, retenons le nombre important de poteries grises de forme tronconique appelée parfois "atrébates " ${ }^{48}$. Des tèles à déversoirs

46 Ce cours deau devirnt an Moyen Age le Merdanson. Voir fig. I sur l'écoulement de la nappe phréatigue.

17 Date de la monnaie la plus tardive : 375 (Gratien, Atelier de Lyon, inv. no 67).

48 L'aide de. M. Klein Bardaggi sur le chantier en 1969) a été précieuse el nous le remercions bien sincerement ici. En 1971 nous avions atlire l'allention des fonilleurs sur l'aire d'expansion de celle céramique (La céramique grise dile d'Arras, dans Archologie, 1971. p. 54-5̄s; La découterle d'une officine de poliers à Labuissiere (Pas-de-Calais),

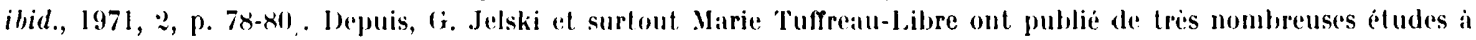
propos de ce materiel. La produclion leauvaisienne a dé publiée avec minulie par J. el F. Canten, Puils gallo-romain, rue du Pressoir-Coquel, dans Ogam, n's 1:21-1:26, 1969, Beauvais. Bien que ceci ne soit pas l'ubjet direct de nos propos, mentionnons cependant la decouverte dans les remblais du sondage 6 de tessons mérovingiens avec decors a croix potences et a motifs animaliers tite de chevaux ou de cerf comme ceux de Vaule). 


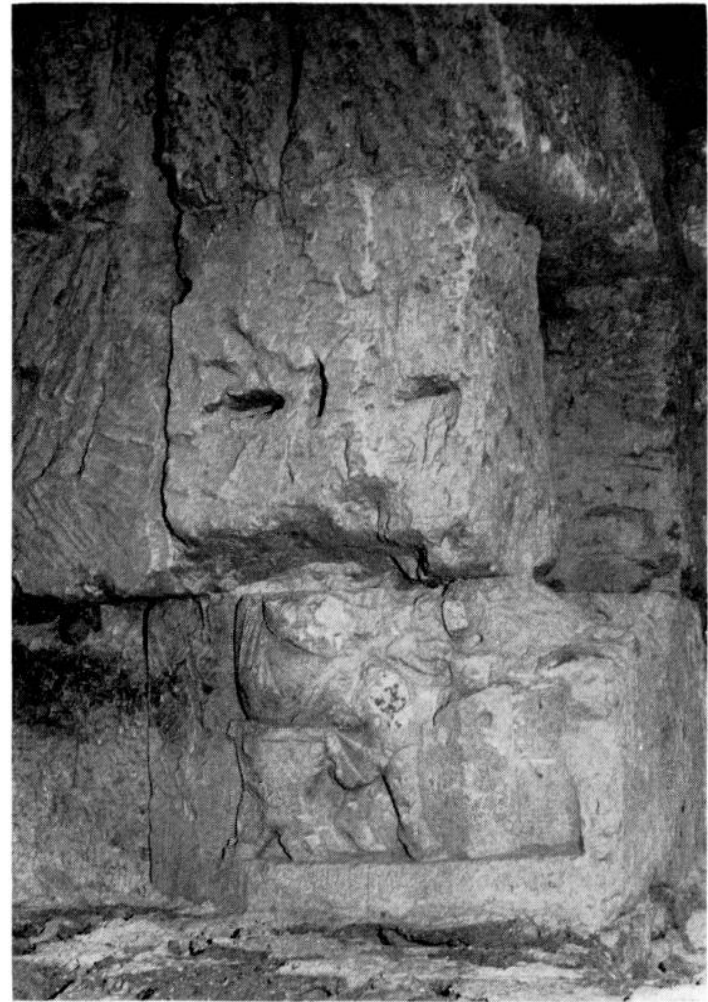

93 Stele funerateremployed dams le rempart.

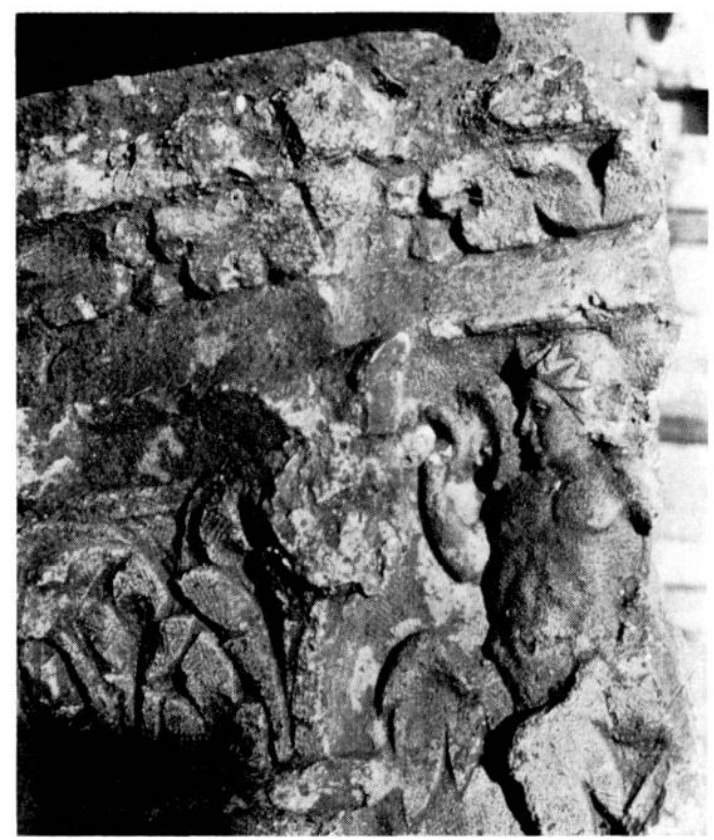

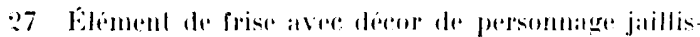

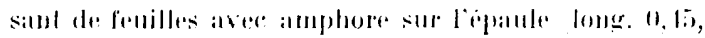
latro. (1, 111, ha111. 01,15 III.

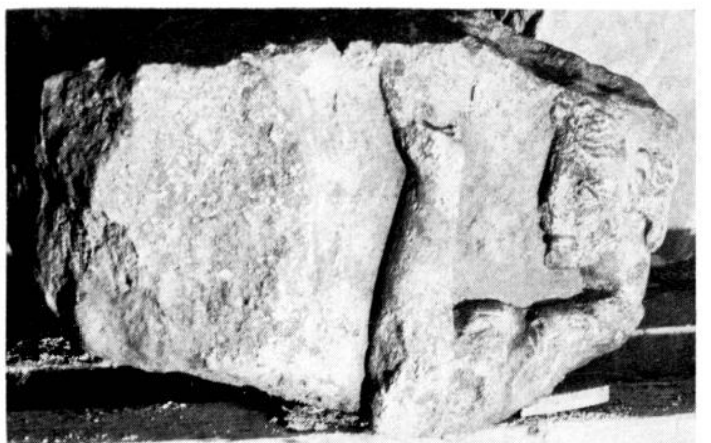

21 Bloc sculpte. Fragment do busle de Minade long.

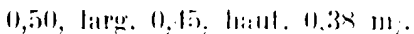

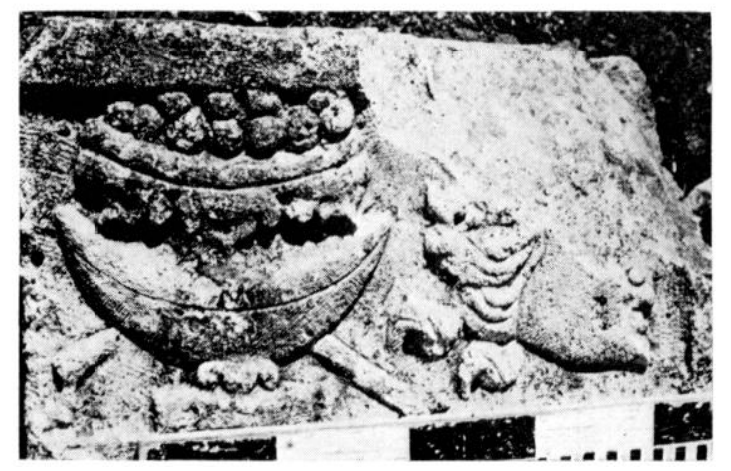

: B Bloc sculpli. Corbrille de fruits et tête féminine long. (1,5s, largr. 0,12, haut. 0,5l m:

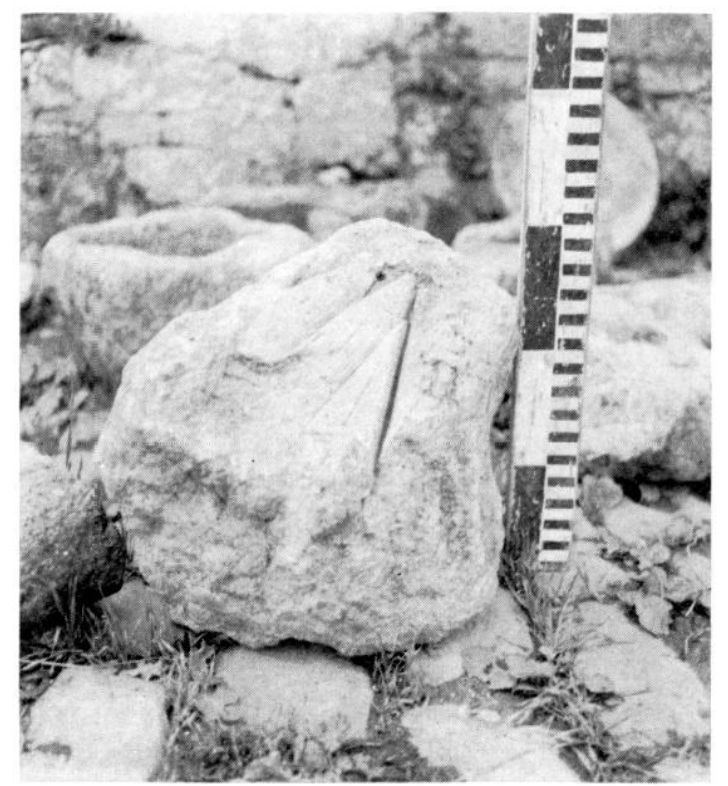

$\because$ Firigment diajte? 
presentent plusieurs variétés de profils el de dimensions. I en juger par la taille du bee verseur. certaines sont de tailles imposantes (près de 5) am de diametre) (fig. 19).

Malériel divers. Des objets en os, retenons un marteau. une rouelle al des alènes (lig. 20). En plusicur's endroits du chantier, des fragments d'enduits peints ont été découverts. Ln seul supporte un décor imagé (lig. :21). Les objets sont représentés soit par des éléments isolés (maillons de chainnes. ustensiles, plaque de coffre au décor de Méduse). soit par des petites statuettes (petit Mercure) ou des éléments de trépied (Vénus casquée. fig. 22). Plusieurs terres cuites (de l Allier ou du liassin parisien) ont ete retrouvées (2 fragments de Vénus anadyomène, une pretite tête, une patte de cheval).

Eléments sculplés. Pres de 60 éléments d'architecture ont dé découverts. Nous poutrons les regrouper en trois séries : élements de colonnarles, lasese et füts (nus ou cisclés); vestiges d'arehiterture monumentale (frise, architrave), éméme d'arehitecture funéraire (piédestal, stele). A part les bordures of les dalles en marbre hlance, toutes ces pierres sont en calcaire local. Elles ont été découvertes soit dans le noyau de la fondation de l'enceinte, soit dans la masse des remblais qui ont recouvert les ruines de la ville du Haut-Empire. Comme nous l'avons vu plus haut, la disposition régulière de blors d'arehitecture laisse supposer la presene d un grand monument central transformé en carriere interne; tandis que les nécropoles limit rophes a la ville fournissaient le complément de pierres aux battisseurs de l'enceinte. L'examen des parois de la cave aménagée sous la tour dite de saint-IIilaire mot en evidence cette double origine; nous y voyons en effet un fragment de stèle funéraire (déja repérée all $\mathrm{xvII}^{\mathrm{e}}$ s., fig. 2:3), el deux éléments de frise monumentale à décor géométriquet9. Deux grands blocs sculptés représentent l'un un fragment de buste féminin. l'autre une corbeille de fruits ot une tête féminine (figr. I4 et 25) ${ }^{50}$. (On retiendra également un fragment d'aile (?) (fig. 26) et un élément de frise avec décor de personnage jaillissant de feuilles aver amphore sur l'épaule (fig. :27). Lin comparant ces éléments avec ceux découverts par Emile Chami au niveau de la Basse (Eure lapin grapillant des raisins) nous pouvons émettre l'hypothese de la présence d'un temple à décor dionysiaque ou d'un autre monument forléon, théatre) illustré à partir du mème répertoire. Il s agit par le style, par le choix de lïronographie, d'éléments que nous pouvons dater de la deuxième moitie du

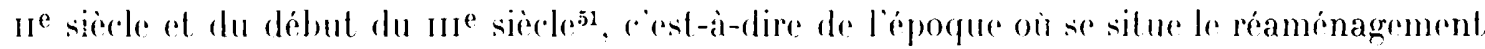
de linsula sous forme de grande plare semi-circulaire. Tout rela avait-il un rapport avee la pièce d'eau situé dans laxe central de cette pièce?

Pierre Lamax

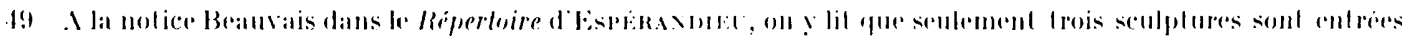

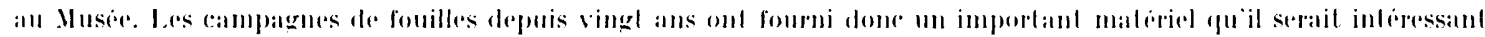

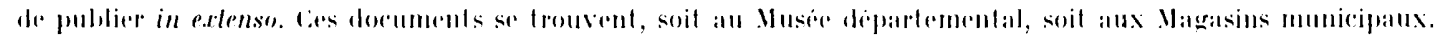

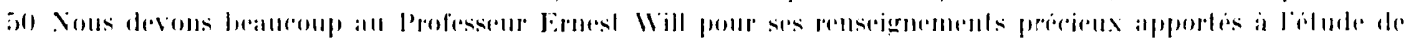
aes dorcuments lappidaires.

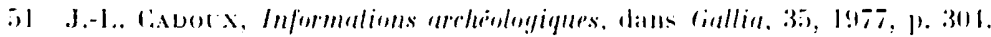

